Emerging Modalities for Soil Carbon Analysis: Sampling Statistics and Economics Workshop

Workshop Summary

\author{
To the \\ DOE/OBER/TCP and \\ DOE/FE/NETL \\ Program Managers
}

Lucian Wielopolski

April 2006

Brookhaven National Laboratory

Upton, New York 11973-5000 
BNL-75762-2006

FORMAL REPORT

\title{
EMERgING MOdalities fOR SOIL CARBon ANALYSIS: SAMPLING STATISTICS AND ECONOMICS WORKSHOP
}

\author{
Workshop Summary \\ To the DOE/OBER/TCP and \\ DOE/FE/NETL \\ Program Managers
}

\author{
Submitted by \\ Lucian Wielopolski, Workshop Organizer \\ Brookhaven National Laboratory \\ Environmental Sciences Department \\ Upton, NY 11973
}

January 19-20, 2006

Lucian Wielopolski Ph.D.

Brookhaven National Laboratory

Environmental Sciences Department

Bldg. 490D

Tel: (631) 344-3656

Upton, NY 11973

Fax: (631) 344-7244

E-mail: $\underline{\text { lwielo@bnl.gov }}$

Acknowledgement: Author expresses special thanks to Avril D. Woodhead for assisting in preparation of this report. This workshop was performed under the auspices of the U.S. Department of Energy, Office of Science, Biological and Environmental Research, Climate Change Research Division and Office of Fossil Energy, National Energy and Technology Laboratory, Contract No. DE-AC02-98CH10886 to Brookhaven National Laboratory. 


\section{DISCLAIMER}

This work was prepared as an account of work sponsored by an agency of the United States Government. Neither the United States Government nor any agency thereof, nor any of their employees, nor any of their contractors, subcontractors or their employees, makes any warranty, express or implied, or assumes any legal liability or responsibility for the accuracy, completeness, or any third party's use or the results of such use of any information, apparatus, product, or process disclosed, or represents that its use would not infringe privately owned rights. Reference herein to any specific commercial product, process, or service by trade name, trademark, manufacturer, or otherwise, does not necessarily constitute or imply its endorsement, recommendation, or favoring by the United States Government or any agency thereof or its contractors or subcontractors. The views and opinions of authors expressed herein do not necessarily state or reflect those of the United States Government or any agency thereof.

Notice: This manuscript has been authored by employees of Brookhaven Science Associates, LLC under Contract No. DE-AC02-98CH10886 with the U.S. Department of Energy. The publisher by accepting the manuscript for publication acknowledges that the United States Government retains a non-exclusive, paid-up, irrevocable, world-wide license to publish or reproduce the published form of this manuscript, or allow others to do so, for United States Government purposes. 
The Department of Energy (DOE) operates Brookhaven National Laboratory (BNL) with its many unique research facilities and programs. One of the DOE's major strategic missions is the Terrestrial Carbon Processes Program that studies the global production and transport of anthropogenic $\mathrm{CO}_{2}$ and its effects on climate and health. BNL's Environmental Sciences Department responds to this mission and the research of Wielopoloski's group in the Environmental Research and Technology Division are closely tied to this goal. Through new instrumentation, focused measurements, and modeling, they aim to determine the in situ carbon content of soils, locally and over large tracts of land. This research, though still in its early stages, is very promising, offering levels of precision not previously attainable, along with speed and lower costs. This meeting will bring together experts in instrumentation and field measurements from other DOE laboratories, universities, and the United States Department of Agriculture to evaluate and explore the novel methodologies and to assess their advantages over conventional methods. 
Table of Contents:

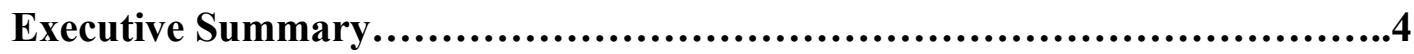

Workshop Rationale..........................................................6

Presentations Summary.........................................................7

Opening Statement...........................................................7

Session I - Instrumentation..............................................9

Session II - Current Soil Sampling Practices..................................14

Session III - New Standards...............................................18

Round Table Discussion....................................................23

Summary...........................................................................28

Appendix A - Agenda Outline....................................................32

Appendix B - Abstracts and Presentations..................................... 34

Appendix C - List of Participants..............................................60

Appendix D - Presentations....................................................664 


\section{Executive Summary}

Terrestrial carbon is an integral part of the global carbon cycle and plays an important role in soil quality and productivity. In the last 20-30 years detailed knowledge of carbon balances and transport in the soil, on local, regional, and global scales emerged as being critically important for quantification of soil carbon stocks. Those are needed for assessing sequestration as a mitigating factor for the anthropomorphic emissions of $\mathrm{CO}_{2}$ into the atmosphere that result in global warming. Lack of reliable instrumentation for monitoring belowground carbon processes and occurring changes exacerbates and hampers implementation of trading policies with carbon credits. With the ratification of Kyoto Protocol, although United States is not a signatory, extensive effort is being devoted to the implementation of strategies reducing the emissions of, and increasing the sequestration of some greenhouse gases, including carbon dioxide. The Intergovernmental Panel on Climate Change (IPCC) recognized that agricultural land and forestry offer the potential to sequester from the atmosphere about 80 billion metric tons of $\mathrm{CO}_{2}$ in the next 50 years. It is self-evident that success of these efforts strongly depends on robust instrumentation for measuring, monitoring, and verifying the processes involved. However, belowground processes pose a special challenge because underground structures, organisms, and processes are invisible to humans. Soil is a dynamic, living system, an intimate mix of living and dead plant matter, fungi, macro- and micro-fauna, plant exudates, and animal waste products, embedded in a matrix of solids, liquids, and gases. When the "black box" of this soil mélange is dissected its function is altered. Current analytical methodologies are not on par with the emerging needs for a fast, reliable, in situ, non-destructive, static/dynamic, and inexpensive instrumentation.

A workshop to explore issues cardinal to the emerging analytical technologies, their operations, and the degree to which they satisfy the needs for an in situ, non-destructive soil carbon analysis was organized by Lucian Wielopolski and held at Brookhaven National Laboratory (BNL) on January 19-20, 2006. It consisted of seventeen presentations and was attended, by invitation, by 30 scientists from the United States and Canada that included universities, industry, other government agencies, and the DOE's national laboratories. The collective expertise represented the fields of instrumentation, soil- and agricultural-sciences. On the first day the technologies presented included Laser Induced Breakdown Spectroscopy (LIBS), Mid- and Near-Infrared Spectroscopy (MIR and NIR), Pyrolysis Molecular Beam Mass Spectroscopy (Py-MBMS), and Inelastic Neutron Scattering (INS). After presentations of the methodologies, current concerns and practices of infield sampling were discussed, followed by delineating the needs and expectations from the new technologies as well as the requirement for establishing new standards or standard sites for instrument evaluation. The second day consisted of round-table deliberations on the quality of the instrumentation, the need for standards, and for independent cross-validation. The entire workshop was audio- and video-recorded.

The goal of this report is to provide a succinct summary of the various technologies that were brought together and to elaborate on the main points that transpired from the workshop. It was widely recognized that the instrumentation presented is based on different technologies that measure substantially different volumes, ranging from few millimeters cube for LIBS, to a fraction of a cubic meter using INS. In addition, it became apparent that LIBS and INS measure 
atomic carbon whereas NIR, MIR, and py-MBMS are sensitive to chemical speciation thus providing different and complimentary information. All the methods indicated existence of some peak interferences and matrix, e.g., soil density and moisture, effects on the measured analyte that require further clarifications and quantification. Error propagation in the measurements was not clarified adequately and it ties in with what constitutes a proper calibration for each instrument. Since there are many ways to calibrate devices, the need became apparent for a standardized procedure or a protocol that would be used at identified sites for independent calibration and double-blind evaluation of the devices. All of the introduced technologies were improving soil analysis by either simplifying it or providing additional information not available with standard soil coring or excavation. The INS method stood apart from the other systems in that it was truly non-destructive, measured large volumes, and it rests upon an analytical response function that enables simulations and synthetic calibrations. However, more detailed information and characterization of all of the systems is required, as discussed in the summary. Nevertheless, it was strongly felt that all methods reached a degree of maturity to warrant joint studies to ascertain the characteristics and performance of each technology, and to crosscorrelate the results among themselves, and with the current standard method of analysis. 


\section{Objectives:}

\section{Workshop Rationale}

The workshop's main objectives are 1) to present the emerging modalities for analyzing carbon in soil, 2) to assess their error propagation, 3) to recommend new protocols and sampling strategies for the new instrumentation, and, 4) to compare the costs of the new methods with traditional chemical ones.

\section{Background:}

Today, the "gold standard" for analyzing elemental carbon in soil is the traditional method of first obtaining a sample by excavating or coring, followed by wet, or preferably, dry combustion. The steps involved in the subsequent chemical analysis are labor-intensive, timeconsuming, and costly, thereby limiting the number of samples used for so-called comprehensive field representation. The problems are particularly acute when the findings from large, nonhomogenous fields are extrapolated regionally and globally. In addition, the destructiveness of this methodology hinders the ability to continuously monitor changes in the soil's carbon stocks for trading purposes, as does its lack of sensitivity in measuring small changes over relatively short periods.

Operational limitations and high costs are significantly reduced by four emerging technologies for analyzing soil carbon in situ. Furthermore, one of them is non-destructive, and can be operated in a scanning mode. However, they exhibit notable differences in calibration and sensitivity, and, importantly, each one samples a different soil volume. Thus, Light Induced Breakdown Spectroscopy (LIBS) analyzes about $1 \mathrm{~mm}^{3}$, Near Infrared (NIR) and mid-Infrared (MDIR) range from 0.5 to $2 \mathrm{~cm}^{3}$, and Inelastic Neutron Scattering (INS) samples above $0.3 \mathrm{~m}^{3}$. These methods may well be best suited for different purposes that complement each other for better understanding of the below ground processes.

The capabilities of the new technologies and their associated errors may change the paradigm of the existing sampling protocols, thus posing the questions: What should be the appropriate one(s) to use and under what particular scenario? How do we demonstrate their special advantages and further their widespread acceptability? How do we devise sampling schemes and reference sites for monitoring carbon changes and stocks at a desired confidence level?

Thus, the tasks of this workshop are threefold: 1) to review the emerging technologies, with particular regard to the instrument's physics (how), sampled volume (where), error of the measurement, calibration errors, biasing, and the sampling schemes, 2) to review the classical description of in-field variability and its ramifications on the new technologies, and, 3) to develop recommendations and guidelines for new sampling protocols that incorporate geostatistical considerations.

\section{Deliverables:}

1) Abstract book for the workshop.

2) Full report of the presentations, discussions, and recommendations.

3) A comprehensive manuscript to be submitted to a reviewed journal. 


\section{Presentations Summary}

\section{Opening Statement:}

The traditional "gold standard" for analyzing elemental carbon in soil is by wet and dry combustion of core samples. The method is cumbersome, labor-intensive, costly, and fraught with approximations due to complexities of the soil matrix and the variability in sampling and analytical procedures. These drawbacks are magnified when the results must be extrapolated regionally and globally for estimating carbon stocks and when restricted field representations are used to assign changes in carbon for credit purposes. Furthermore, the destructiveness of the methodology hinders the ability to meaningfully monitor carbon sequestration and the belowground processes.

Five emerging technologies that potentially offer improvements in assessing soil carbon in situ may significantly reduce these limitations, lower the costs, and add a new insight to the belowground processes: Laser Induced Breakdown Spectroscopy (LIBS), Near Infra-Red Spectroscopy (NIR), Mid Infra-Red Spectroscopy (MIDR), Pyrolytic Molecular Beam Mass Spectroscopy (py-MBMS), and Inelastic Neutron Scattering (INS). Presently, their capabilities and the advantages they offer are incompletely documented.

In response to one of the Department of Energy's (DOE's) strategic missions, The Terrestrial Carbon Processes Program, which explores the global production of carbon and its effects on climate and human health, the Environmental Sciences Department at Brookhaven National Laboratory (BNL) decided to host this workshop to evaluate these novel technologies and compare them with conventional methods. Furthermore, our discussions will be of high importance to the work of the National Energy Technology Laboratory (NETL) charged with meeting a second DOE goal to protect national and economic security by promoting diverse sources of reliable and environmentally sound energy; in particular, their research on monitoring, mitigation, and verification requires the development of novel precise instrumentation. The meeting, organized by Lucian Wielopolski, and attended by experts in instrumentation and field measurements, had three main objectives: 1) To review these novel technologies, especially their basic physics together with their biases and the errors in measurements and calibration, 2) to review the current practices in light of the in-field variability and its ramifications on the old and new methods, and, 3) to develop recommendations and guidelines for sampling protocols for the new instrumentation that incorporate geostatistical considerations.

Creighton Wirick, Chairperson, BNL's Environmental Sciences Department, welcomed participants, and gave background information on the Laboratory, its major researches, its Directorates and Divisions, funding sources, and accomplishments. He focused on BNL's expertise in designing and developing special instruments, particularly those used in the 1990s to take a global inventory of carbon in the oceans, and those used in the DOE/BER's four FACE facilities. Further, BNL's staff are fully conversant with calibration and standardization having, amongst other work, calibrated the satellite instruments for NASA's atmospheric radiation program. Hence, he considered it appropriate to host members of the soil community here to collectively generate ideas for assessing carbon distribution worldwide.

Roger Dahlman, Program Manager, Terrestrial Carbon Sequestration Research, DOE/BER's Office of Science, strongly supported the value of the broad base of expertise at the 
workshop since accurate measurements of soil carbon undoubtedly underpin the concept of sequestration and allotting carbon credits, mentioning in this context, the unresolved attempts to evaluate carbon sinks. In an opening series of projections, he outlined the DOE's support of research in terrestrial, oceanic, and geological sequestration, and functional microbic genome-tolife sequestration. He touched on several sobering concerns, including the role that new instruments might play once their fundamental operational parameters and limitations were known, how they might predict the effectiveness of changing agricultural practices on sequestration, and the need for good databases backing up the research. He concluded with the hope that the workshop might change some present-day perspectives on these problems.

John Lytinski, Program Manager, DOE/FE/NETL echoed the sentiments expressed by Dahlman and explained briefly the specific role and interest that his department has in the new instrumentation. The NETL manages a portfolio of laboratory and field R\&D focused on technologies with the potential for reducing greenhouse gas emissions and controlling climate changes. They need instruments that will accurately account for stored carbon, with a high level of confidence that it will remain sequestered. The instruments must precisely measure carbon, generating results that are easy to interpret and analyze.

A brief discussion of the meeting's objectives by Lucian Wielopolski followed, principally centering on what exactly is being measured, how, and where, and also on how the new methods differed from the conventional wisdom of assessments by core analysis. He stressed that participants should collectively formulate recommendations and standards for carbon measurements.

FOOTNOTE: The following synopses of the formal presentations are expanded in abstracts contained in the appendix to this document. The slides and illustrations accompanying each talk are included on a separate CD and the entire workshop can be seen on http://www.bnl.gov/sca/. 


\section{SESSION I - Instrumentation}

\section{Session Chair, John Litynski, DOE/NETL}

The first presentation, Laser-Induced Breakdown Spectroscopy (LIBS), given by Ronny Harris (co-author Michael H. Ebinger), described this field-deployable high-resolution instrument in which a 1-mm spot laser beam focused on a pressed soil sample rapidly induces the production of a plasma that, in turn, emits a characteristic spectrum with a high density of elemental information. His slides detailed the portability of the instrument in the field, its ability to derive multiple measurements from a small soil volume, and the resolution of the analysis. By dividing core samples, detailed profiles of carbon content can be obtained at various depths, with each individual reading, comprising yield from 10 spectra, requiring only a one-second time scan. Harris described how homogenous samples of different textured soils, viz. loam, clay, and sand, were compressed into flat 30-mm disks and good calibrations curves obtained for each individual one in plotting mean peak area for carbon against the dry combustion percentage of carbon. Interestingly, it recently was shown that the separate calibrations could come together to form one line, thereby simplifying and standardizing the procedure. Their researches showed that the relative standard deviation (RSD) on the mean value decreased with the increasing number of laser exposures, with an acceptable number being obtained after 30 shots (RSD 3-5\%); thereafter, there was a diminishing return in precision up to 100 shots, although many may have to be taken when samples differed radically.

The portable instrument's level of detection (LOD) was better than $0.1 \%$ carbon (300ppm); it costs $\sim \$ 30,000$, depending upon the detector used. Presently, it measures organic and inorganic forms of carbon, but by relating them to changes in the signal from magnesium, they might be differentiated. There are problems remaining in three areas: 1) Overcoming the effects of the soil's matrix, i.e., the presence of roots and grit in the cores that must be removed and the soil homogenized, 2) Insuring adequate sampling of a field, since the samples are small and many more may be needed, and, 3) Calibrating the method in-field, as opposed to today's calibration with dry combustion methods.

The ensuing discussion, in common with all that followed each presentation, was vigorous, and occasionally controversial, as anticipated and hoped for in attempting to resolve the issues facing the soil community. We do not distinguish individual contributions to the discussions, but give the gist of the questions and responses.

Here, the questions revolved around the variance in the values, how to express the variance, and whether to remove non-sequestered carbonaceous material (surface leaves). Harris pointed out that if the samples were very heterogeneous; more shots were taken although this involved a great deal of data. However, he mentioned that the sample began to burn up after 60 shots. Further, he stated that non-sequestered, but rotting leaves could be distinguished as spikes in the spectra. In replying to a question on the effects of the soil's variable moisture content, he said that it was not a major drawback as the soil could be dried in a few seconds in the field.

Colin Christy, Veris Technologies, next discussed In-Situ Measurement of Soil Carbon by Near Infrared Diffuse Reflectance Spectroscopy. This valuable in situ technique beams nearinfrared light into the soil where it is absorbed by molecular vibrations, the harmonics of which 
are recorded and measured. Not all are measured, primarily those with strong bonds, C-H, N-H, and $\mathrm{O}-\mathrm{H}$, useful for quantifying carbon, nitrogen, and water, respectively, and secondarily, those with $\mathrm{C}-\mathrm{O}, \mathrm{S}-\mathrm{H}$, and hydrogen bonds. The underlying physics laws are those of diffuse reflectance (nonzero path - quasi Beers Law). Kristy showed diagrams of the instrument's configuration in acquiring probe-based- and shank-based- measurements, along with the resulting absorbance spectra. The instrument can be affixed on tillage equipment, and moved over the field at four- to six-mph with the sapphire window (excellent optical properties) in direct contact with the soil, collecting the diffuse reflectance from between 5-to15-cm below the surface. The probe, pushed into the soil, measures vertical profiles down to $30-\mathrm{cm}$ or more. In calculating the light absorbed, three measures are obtained; a dark spectrum (no light for 5-10 minutes), a spectrum from reference material (5-10 minutes), and finally, the sample's spectrum. In an example of absorbance spectra from 204 locations, water is evident as a large clearly visible peak at a wavelength of $2000 \mathrm{~nm}$. Other changes are subtler, and chemometric methods are used to construct models relating the spectra to particular elements. The many data are compressed, expressed as Principal Component (PC) scores, and mapped. Also, cluster analysis determines the samples' locations, so that one can be taken from each cluster to give a good representation of the soil's variability.

Kristy next illustrated in his slides their work on calibrating and validating the method in which they had explored many parameters, removed some data to see how the information was affected, so that they could judge how many samples were needed. In field applications, they found that the spectrum might not be reliable when there is an outlier to the calibration population. Overall, the advantages of the method are that it is fast, no sample preparation is needed, many parameters are measured at once, and water, often problematic, is a low absorber. On the other hand, samples and analyses are required for calibration and validation, and the computations involved are intensive.

Questions concerned whether the water spectrum had to be subtracted from the data; Kristy replied that this does not get in the way. In response to queries about variability, he replied that they take thousands of spectra in analyzing a field to characterize its elemental components, assign it to a group, and develop databases for different locales, rather than using databases from different areas. The possibility was raised of expressing the findings in percentages of the main chemical bonds.

The third presentation, DRIFTS Diffuse Reflectance Infrared Fourier Transform Spectroscopy, was given by James Reeves, USDA (co-authored with Gregory McCarty). DRIFTS, originally a laboratory-based system, irradiates soil samples with mid-infrared radiation, and collects the diffusely reflected radiation. Up to 360 samples a day can be analyzed, compared to 100 by traditional combustion methods; recent developments resulted in a portable system, one foot tall and weighing 10-15 pounds, with scanning capabilities that can be used in the field. The instrument costs about $\$ 37,000$. DRIFTS rapidly identifies a wide variety of soil carbon fractions, including free amino acids, enzymes, sugars, and charcoal, along with inorganic nitrogen. Reeves illustrated its capabilities in a series of detailed slides showing the range of variation in organic-, inorganic- and total-carbon from 237 soil samples, the effects of acidification of the samples, and the outcomes of several calibrations and validations using 174 samples from fields in Maryland. Reeves discussed their comparisons of calibrations derived for 
soils using near- and mid-infrared spectra that showed the latter was more accurate, robust, with fewer outliers, and easier to interpret than the former. Similarly, in both approaches the presence of inorganic carbon interferes with the determination of organic carbon, but again, the results were best with mid-infrared. However, in samples of grains, there was little to choose between them. Overall, the findings showed variability, and in some cases, even for the same plant grown in different areas, different calibrations might be required. Reeves noted, however, that the socalled "gold standard" also suffered from variability; different combustion units from different manufacturers revealed variations. He finished by pointing out the method's drawbacks, viz., too few samples, improper ones, especially artificial samples with much less variability that natural soils, and the use of surrogate calibrations rather than ones from true sample analytes.

In the following discussion on measuring charcoal, Reeves said that, as yet, the data are not good, even though an Australian company had commercialized a mid-infrared detection system that was being used. With data from the USDA, he found that the charcoal value changed with the treatment's temperature. He agreed that mid-infrared might be useful for assessing the soil's bulk density. He had good results with samples of sand, silt, and clay, and found that particle size had no effect.

Kimberly Magrini, NREL, discussed the characterization of forest, agricultural, and grassland soils in Rapid Identification and Quantification of Soil Organic Carbon Components using Pyrolysis Molecular Beam Mass Spectrometry (with co-authors M. Davis, J. Kimble, R. Follett, C. Hoover. E. Paul, and R. Evans). Using analytical pyrolysis, a twenty-year old technique, with molecular beam mass spectrometry, i.e., py-MBMS, suitably adapted and customized for work with soils, (for example, by changing its combustion mode), they explored its breadth of applicability in analyzing, and grouping the principal components of soils from several locations and depths, under different management practices, and containing litter and roots. Multivariate statistical analyses were used to quantify the results. Her illustrations showed the set-up of the triple quadrupole MBMS in the laboratory, which was transportable, but not suitable for field use. The following slides showed its good performance in distinguishing between undisturbed and disturbed soils (after a tornado), between new (shallow samples) and older carbon (deeper samples), such that the fingerprint of aromatics in the old one, revealed by principal component analysis, can facilitate grouping as a function of depth. It further revealed differences in the ratio of new and old carbon with depths, from $0-5 \mathrm{~cm}, 5-15 \mathrm{~cm}$, and $15-30 \mathrm{~cm}$, based on parent compounds, and, in poplar forest soils, it was useful in separating top leaf litter, deep root litter, and litter from stems. It also served as a biomarker for microbial biomass (SMBC). The authors presented six slides of regression coefficients for estimating various soil fractions, including those for particulate organic matter, POM, and carbon mineral matter, and also one of soil organic carbon against calendar age. The instrument proved useful in demonstrating the effects of different land management practices at a site in Idaho, particularly its potential for rapidly quantifying, in 3-5 minutes, SMBC, C-min, and POM C in samples of $100-500 \mathrm{mg}$. Future work will develop data-based multivariate models to assess the pathways of carbon sequestration, resolve fundamentals of the chemistry involved, and will consider ecological questions. In replying to a question about the value of the structural information obtained compared with solid-state NMR and the like, Magrini stated there are differences in the way that the samples are ionized and she considers the technique as complementary to others. 
Lucian Wielopolski, BNL, next presented Soil Carbon Analysis by Inelastic Neutron Scattering, (co-authors S. Mitra, O. Doron, and Y. Zhang). In describing Inelastic Neutron Scattering (INS), an in situ non-destructive field scanning approach to detecting carbon in soil. Wielopolski first posed questions on what, how, and where is being measured; then he outlined its advantages over traditional methods. INS is based on gamma-ray spectroscopy induced by fast $14.0-\mathrm{MeV}$ neutrons impinging upon elements in the soil. In addition, fast neutrons undergo elastic collisions, which after multiple scatterings, slow down to thermal energies, and are captured by the soil's elements further inducing the emission of gamma rays. Total carbon can be measured to $\sim 25 \mathrm{~cm}$ deep, with a footprint of $\sim 2.3 \mathrm{~m}^{2}$, and a volume of $\sim 0.3 \mathrm{~m}^{3}$. He showed representative spectra and the related basic definitions for calculating the net peak intensities, discussed two major spectral interferences with the carbon peak, including the effects of silicon, and described how to identify the area of interest, calculate and deduct the background, and express the findings in various ways.

Wielopolski next described his initial calibrations of a prototype instrument in a pit, $5 \times 4 \times 1.5$-foot, of hand-mixed synthetic soil; a good curve for carbon concentration $(\mathrm{gC} / \mathrm{cc})$ was obtained after correcting for silicon interference. Similar successful experiments then were carried out at BNL in a pine stand, oak forest, and sandy soils, and later in Duke Forest, North Carolina, in which the problem of outliers in the data were resolved, such as those reflecting the presence of charcoal in the soil, before re-plotting the calibration curves. A newer self-sustained version of the INS system, mounted on a gantry, was tested recently in soggy clay and sandyloam Alabama soils. Here, up to $20 \%$ variability was recorded in five cores from one square foot of soil.

The speaker next gave details about the analytic model of the system, derived from basic principles and fundamental parameters, including the detector's response functions, as well as the soil's variability. Explicit functions were revealed and illustrated in the slides. Similarly, Monte Carlo simulations generated a conceptual model of the system, and allowed calibration to be created for an arbitrary soil and soil conditions. In summary, the INS system offers advantages as a non-destructive in situ large-scale scanning approach that can provide an integral count of total carbon down to $\sim 25 \mathrm{~cm}$. Future research will concentrate on increasing the system's sensitivity, establishing calibrations to measure total carbon levels and changes in them, and validating and cross-correlating the findings with field studies. Questions following the lecture centered on the differences between the results from the INS and those from chemical analyses. Wielopolski pointed out that his findings represent integral values of the total carbon encountered in the volume seen by the detector. Asked whether a calibration would be needed for each field, he stated that the chemistry of the soil does not affect the total carbon counts, thus he hopes to generate a universal calibration for the method.

The Common Approach for Measuring Soil C and Soil C Sequestration was the subject of the last presentation of the session, given by Achim Dobermann, University of Nebraska. His focus was to draw attention to the drawbacks of the standard classical method of analyzing carbon, against which the new technologies are measured. He first outlined, in illustrations, the six steps in the classical approach to assessing soil carbon, viz., collecting field samples, processing them, measuring $\mathrm{C}$ mass fraction, calculating the total amount of $\mathrm{C}$ for a certain depth 
or soil mass, and determining the change in $\mathrm{C}$ or its sequestration rate. He detailed the many sources of variability in each step that 1) for sampling included the season, number of samples, their depth, and the bulking strategy employed, 2) for processing included drying, removing debris, and grinding the sampling, and, 3) for measurement included the gold standard, older researches, routine soil testing, and the emerging methods. His graph plotting the deviation from the average \% C in 24 samples ( 3 runs of each sample) analyzed by dry combustion at five laboratories showed marked differences between them, and in one laboratory, the repeated measurements were very different. He illustrated more problems in repeatability, with big differences due to the mesh size used in grinding that had major implications in predicting and mapping carbon stocks.

The "gold standard" expresses $\mathrm{C}$ on an equivalent dry soil basis, but in most reports, soil volume is used that is satisfactory only if the soil's densities are the same, or if the samples are deep ones. He compared constant soil depth with constant soil mass and proposed that the better measure is expressing $\mathrm{C}$ on a dry mass basis. He explored the problems of how to express sequestration as an absolute value over time, since most data are relative. A comparison of values on a relative basis from a no-till field and a chisel-plowed field revealed that expressing $\mathrm{C}$ as constant soil depth suggested sequestration, but expressed in terms of soil mass, carbon was being lost. Often, expressing carbon as constant mass gives values $40 \%$ less than when expressed as constant depth. He also cautioned about using early studies in which the errors could add up to misjudgments about carbon sequestration. He suggests that less carbon is being sequestered than thought, but suggests that measurements should be made at landscape levels rather than field levels.

Members of the audience agreed with his conclusions on not using soil density, but considered that relative comparisons are useful. It was pointed out that both climatic changes and management practices could entail absolute changes in carbon. It was agreed that knowledge of both would be very useful. 


\section{SESSION II - Current Soil Sampling Practices}

\section{Session Chair, Nancy Cavallaro, USDA}

The session began with a presentation given by Cesar Izaurralde, and co-authored by Allison Thomson, and Aaron Rappaport (Joint Global Change Research Institute) entitled, Measuring and Upscaling Soil Carbon Changes. Izaurralde commented first upon three projects in the 1990s, from industry, the private sector, and the Canadian government that centered on the possibility of upscaling measurements of sequestered carbon from field to regional levels, and highlighted the challenges involved. He focused on the field level, where there are manifold problems in developing cost-effective measures to detect carbon changes related to management practices. He then outlined some of the direct and indirect methodologies used, the problems inherent in sampling, laboratory procedures, and calculations, suggesting that upscaling may have to be based on findings from several systems and models, complemented by remote sensors over large areas. Previous assessments have been static; active ones are needed to detect changes in carbon. He mentioned the acute problem of dealing with spatial variability: his recent research showed that 16 samples could only detect a change in soil organic content (SOC) of the order of $10-15 \%$, and 100 samples were needed to detect a $2-3 \%$ change. This variability might be countered by the approach of using specified non-random "microsites" as was done in the Prairie Soil Carbon Balance (PSCB) project that over three years recorded changes of 0.71and $1.25 \mathrm{Mg}$ $\mathrm{C}$ ha ${ }^{-1}$ in semiarid and sub-humid soils, respectively. Further complicating the picture is that the changes are usually small against a background of large soil $\mathrm{C}$ stocks $\left(0.1-0.5 \mathrm{kgC} \mathrm{m}{ }^{-2}\right.$ versus $2-8$ $\mathrm{kgC} \mathrm{m}^{-2}$ ). Also, the packing of the SOC, cubic or dense hexagonal, affects the soil's density and hence, temporal comparisons of $\mathrm{C}$ stocks. Indeed, differences between SOC levels in different countries might reflect cubic versus random packaging. He suggested adopting Time Domain Reflectology (TDR) to measure the soil's density and using a thermo-TDR probe that yields a variety of valuable data; however, improvements are needed in its accuracy and reliability.

The speaker evaluated the models that had been tested against long-term data, and their ability to scale up the findings. SOCRATES usefully modeled carbon fluxes in two ecodistricts in Alberta, applying three scaling up procedures, but was limited by spatial variability and the soil survey data. He touched upon their recent work assessing the impacts of climate change upon agricultural resources and soil sequestration in China; based on 18 selected farms, differences were found between no-tilled and conventionally tilled field with different crops. Notilled fields sequestered more carbon. In summary, methods for measuring and upscaling soil $\mathrm{C}$ changes must be based on the best science, use a system perspective, be verifiable and comparable, and report uncertainties. He was questioned about whether the models were reliable because they were calibrated against measured data that might be faulty, and hence, the models biased. Izaurrade cited the EPIC model the calibration of which is changed annually to address this problem. The data from China had also been corrected to deal with bulk density, and error bars added, and sensitivity analyses undertaken.

Charles Rice, Kansas State University, gave the next presentation, Monitoring Changes in Soil Carbon in the Field. His first slides set out some essential factors in measuring, monitoring, and verifying the soil's carbon content, based on the best science that encompassed 
transparency in reporting, cost-effectiveness, ability to meet the requirements of international conventions, and compatibility with old methodologies, but flexible enough to accommodate new instruments, processes, and models. Associated uncertainties, start and end times, and the mean and variations should be reported. He expanded upon the issues in estimating soil organic carbon, discussing the influence of variability due to seasonality entailing changes in the soil's bulk density, crop rotations, depths, rocks, carbonates, and especially roots. Horizontal (landscape) and vertical variability also must be accounted for; he showed in-field examples of such variability.

Rice suggested that establishing spatially explicit reference sites (geo-reference microsites) across major soil types might lessen these problems. Furthermore, such a simple approach would be cost-effective, and invaluable in monitoring temporal changes, and facilitating the introduction of new technologies. His next seven slides gave detailed results from the sampling protocols used in agricultural and prairie soils, which he compared with the findings from such microsites in the Prairie Soil Carbon Balance (PSCB) project. He discussed grid spacings and the effects of sampling at different depths. The coefficient of variation \% was greatest in the topsoil and higher where the soil was tilled, but less deep down; variations in the soil's bulk density were a large source of error. A major conclusion was that random resampling could not detect small changes in soil carbon over less than five years, whereas repeated sampling at defined microsites (PSCB) lowered the minimum detectable difference. Using ancillary information, models, along with destructive sampling, further increased accuracy in assessing soil carbon. In response to a question about the variability introduced by field and laboratory workers, Rice suggested that standard training for operatives and inter-laboratory comparisons were needed.

The following presentation, Strategies for Monitoring and Mapping of Soil Carbon in Large Fields, was given by Achim Doberman, University of Nebraska. His first slide, giving the typically large total amount of soil organic carbon (SOC), $165 \mathrm{Mg} \mathrm{C} / \mathrm{ha}$, in soils in eastern Nebraska, demonstrated the difficulty of detecting small sequestrations, even over five years (2.5\%). He described issues in optimum sampling and mapping strategies for creating maps of SOC, as exemplified in their researches in three large fields in the Carbon Sequestration Research Facility in Mead, Nebraska. Between 200-250 core samples from three sites, 0-30cm deep, showed major differences in carbon content, with CVs of 17-26\%. His classical estimator of the sample size required showed that twenty composite sample per field, with 20 sub-samples in each, lowered the mean detectable difference to $2.5 \mathrm{Mg} \mathrm{C}^{-1 \mathrm{ha}^{-1}}$ with a $90 \%$ confidence limit. In a series of calculations he showed how correlating the SOC with spatially dense secondary data, such as electrical conductivity, reflectance, models, and bare soil imagery greatly increased accuracy, as demonstrated when two of these parameters were put into a regression estimator. This lowered standard error, so that fewer samples were needed, and hence, reduced costs. The major implication of his findings was that random re-sampling cannot detect small changes in carbon in less than five years, but geo-referenced paired sampling can. He touched upon the value of random composite samples, combined with the regression estimator, which can reveal changes in carbon between 5-10 years. He found regression kriging (synonymous with optimal prediction, it is an interpolation method predicting values at unknown locations from those at known ones) along with secondary data is superior to mapping techniques that use only SOC, 
and reduces the number of samples needed; further, it improves the accuracy of maps 15-35\% compared with ordinary kriging.

Kurt Johnsen, USDA Forest Service, discussed Unique Aspects of Sampling Soil C in Forest Systems. Johnsen's presentation highlighted the extreme challenges in determining the soil's carbon content in forests. Horizontal and vertical spatial variation of carbon in forests is uniquely high because they are subject to many disturbances, as he illustrated in his dramatically different photographs of forests where logging occurred, or where they had been devastated by fire. Further, such marked variation exists even in small apparently homogenous areas, as evident in a map of the forest cover at the Savannah River Site, and in the findings with depth in cores within one stand. Management practices, especially in wet areas after bedding, that displace the soil mechanically, create troughs and mounds; the incorporation of organic matter from leaf litter into the raised beds, or the application of mulch to them, causes partial inversion of the soil's carbon profile (down to $40 \mathrm{~cm}$ below the surface), an effect that persists for up to 20 years after treatment. Troughs in high bulk-density soils typically have the lowest carbon content. Coarse fragments of woody debris and stones impede random sampling and have to be taken into account for proper scaling. Johnsen compared three sites, the "good", the "bad", and the "ugly", the first with no pebbles, the second with rocks large and small, and the last in Duke Forest where the mean volume of coarse fragments is $12 \%$, but with a range of 0 to $48 \%$.

Temporal variations in carbon may also be great, as large roots, or shallow dense mats in forests of black spruce trees, decompose yielding "hot spots" that may still be recorded after 20 years. Radical changes in the soil's moisture content in forests often follow clear cutting: the lack of water transpiration from the trees raises the level of the water table, often resulting in long periods of standing water.

Johnsen concluded that forests exhibit extremely high variations in carbon content because of disturbances, land-use history, and because many are poor sites. Therefore, while non-destructive sampling would be excellent, he believes it would be very hard to implement. He noted that $70 \%$ of the land in the eastern United States is forests.

Harold Van Es, Cornell University, next discussed Sampling Procedures, Sample Volume and Accuracy. Van Es commented that there are many sampling methods, and it is hard to give answers on the field scale. He has experience with Visible Near Infrared (VNIR) reflectance that involves measurement scales of $\sim \mathrm{cm}$ range, as does the MIR reflectance and the gold standard, compared with LIBS $\sim \mathrm{mm}$, and INS $\sim \mathrm{m}$. He gave details of the instrument's underlying statistical model, often assumed to have parameters of a normal Gaussian distribution, or probability distribution function. However, he noted that problems of variability had to be deal with in field samples. Bias may exist in the methodology because of the non-coincidence of the sample's volume and that of the measurement's observed volume/area, such as when glomalin (a protein made by fungi) surrounds soil aggregates, so that the system may "sense" an area that is physically $\mathrm{C}$ enriched or impoverished. Furthermore, if the measurement process is nonlinear (of which he is uncertain for VINS), the mean response may depend on the sample's volume. He pointed out that variance decreases with the scale of measurement so that, for equally precise methods, those measuring small volumes will be less accurate. Van Es next touched on Bear's 1972 concept of Representative Elementary Volume (REV), i.e., the smallest that can represent 
the range of microscopic variations, and illustrated how the sample's volume skews the variability structure and shape of the distribution function; however, despite this drawback, he proposed that since treating smaller samples is less expensive, and considering costs, using many small samples may give a better representation than one large one; he offered a ratio to determine this. 


\section{SESSION III - New Standards}

\section{Session Chair: Tristram West, Oak Ridge National Laboratory}

In his opening remarks, West commented on some essential factors that must be considered in compiling data on carbon sequestration, and in projecting the scale for carbon credits so that they can be assigned on a regional basis. He discussed carbon accounting in soils, fossil fuels, and by remote sensing by surface reflectance, the latter being an active field in scaling up.

Lucian Wielopolski (with co-authors Sudeep Mira, Oded Doron, and Yue Zhang) gave the first talk in this session, A Scanning System for Soil Carbon Analysis. Tying his presentation into West's comments, and first showing four slides, he described the femtosecond temporal sequences underlying the response of the Inelastic Neutron Scattering (INS) system wherein fast neutrons beamed through the soil are thermalized, yielding gamma rays that are recorded in detectors. This non-destructive novel system can contiguously scan large areas, yet, with the tractor carrying the instrument moving at speeds of 4-6 mph, the system is virtually stationary relatively to these rapid nuclear events. His equations demonstrated the kernel of static measurement, so that, during scanning, a mean field response can be obtained from the integral response of the scanning system. He presented a basic functional description of the system and described how Monte Carlo calculations estimate the multiple integrals in the equation and can simulate its response under field conditions.

Wielopolski discussed field experiments that demonstrated the viability of the INS system for scanning. Several Alabaman large fields with clay, silty clay, and loam soils were measured statically and scanned at variable speeds: he showed that the scans differed from the mean of four static measurements in each field in terms of standard deviation (SD) by $0.1 \mathrm{SD}$, $0.8 \mathrm{SD}$, and $0.7 \mathrm{SD}$, respectively. He discussed how their conceptual model simulating a field of $250 \times 200 \times 50 \mathrm{~cm}^{3}$ (represented by $2,500,0001 \mathrm{~cm}^{3}$ voxels), generated a proper system response to layers of carbon, $5 \mathrm{~cm}$ thick, and located at three different depths down to $25 \mathrm{~cm}$. He then moved on to describe the new beta-type INS system with $16 \mathrm{NaI}$ detectors, and considered how, in future, the calculations would be expanded to express error propagation as a function of field parameters, and predict the number of samples or sample size needed for a given confidence level under specified environmental conditions. In response to several questions, he agreed that there were certain terrains, such as forest, where the instrument cannot readily be used for scanning purposes and further, that it records surface litter as well so it must be removed first to properly record the amount of carbon in the soil in such environments. He reiterated that the INS system integrates the result, giving a mean response for an area, and for relative changes does not require calibration.

David Brown, Montana State University, discussed Calibration, Validation, and the Question of Outliers. His presentation, largely on Visible and Near-Infrared (VNIR) and MidInfrared (MIR) diffuse reflectance spectroscopy, explored and offered solutions for the problems of independent validation, outliers, and systematic errors. His work was based upon case studies 
at six sites in Montana with similar soil factors, management, and ranges of properties. Independent validation of soil carbon content obtained from these IR methods against reference laboratory methods is infrequently undertaken; his slides clearly demonstrated that so-called randomly selected "holdout" validation samples taken from randomly selected soil profiles are not truly independent, and illustrated how such samples might be obtained by using random "holdout" validation profiles or by cross-validation by profile. Calibrations from the Montana sites showed how some fields predicted organic carbon values poorly with the infrared methods, suggesting that samples from up to twelve sites may be needed to approach robust results. Calibration statistics are poor compared with validation statistics. Turning next to spectral outliers he showed, from a pair-wise analysis of 4184 samples from a soil-spectral library, the fallacy of the expectation that samples looking similar will have the same composition, and vice versa, so that spectra must be pre-screened. He discussed removing outliers in plots of VNIR predictions against laboratory references, and cautioned about using automatic chemometric packages to remove them. Calibration outliers should be removed only if they are influential, but validation errors must not be removed without independent evidence of error. In analyzing systematic errors, Brown prefers using the root mean squared deviation (RMSD) rather than the Standard Error of Prediction (SEPD) because the former can be partitioned. His slides of VNIR prediction versus laboratory reference showed the ideal 1:1 regression line, one with error due to scatter, one with error due to non-unity slope, and the final one with error due to bias that is the most important one; in summary, he suggested how each could be deal with. Participants suggested that bias does not invariably equate with systematic error, and may indicate other problems, and also pointed to that fact that some of these problems were inherent in the early design of such instruments and cannot now be easily changed.

Next, Reference Materials and Synthetic Soils was discussed by Ben Ellert (co-author H. Janzen). Errors in determining soil carbon content can occur at all stages, with the highest probability during field sampling. Noting that chemical analyses also introduce errors, the standards of the essential reference materials used to validate and calibrate the accuracy of methodologies are critically important. Qualified laboratories must establish and document their materials' composition and properties, ensure traceability, and also a close resemblance between the background composition of matrix reference materials and that of the samples. Ellert's slides displayed worldwide commercial and institutional sources of such materials, and commercial and non-commercial databases on soils. He dwelt upon the difficulty of finding good standard certified materials, particularly for carbon compared with samples for metals. Eurosoil, for example, has seven ranges for organic and inorganic carbon, but only three data sets. In 25 materials, only six had organic carbon and few had nitrogen values. Among soil types, the reference materials for freshwater sediments were the worst. He measured inter-standard consistency in reference soils for carbon, nitrogen, and plant nitrogen, plotting measured values against expected ones and obtained good correlations.

Ellert suggested that reconstructed soils might be valuable in assessing carbon stocks. Such soils typically are used to verify laboratory analyses, and are prepared by fortifying field soils; he suggested that soils reconstructed with a range of carbon content might be used to evaluate novel techniques in situ. He described, and illustrated, a large soil-quality experiment designed to explore the soils' productivity, and to detect temporal changes in carbon; here, the 
topsoil was removed (leaving the same subsoil) and replaced with 36 disparate soils from other areas, and plant growth in each plot subsequently compared. He suggested that a comparable approach potentially might afford a novel way in which to estimate carbon stocks, and evaluate new techniques. In response to questions, he said that this experimental site is still available, though there was concern about using it for destructive sampling.

Paul Doraiswamy, USDA, (with co-authors E. Hunt, R. Izaurralde, and G. McCarthy) next described Modeling Soil Carbon Sequestration and Scaling up to Large Areas. Their ultimate goal in their researches was to help farmers increase sequestration, and obtain credits, so their initial focus was upon assessing carbon stocks in the soil under various scenarios and scaling this up to regional levels; however, because of the enormous inherent complexity and spatial variability in crop lands at regional levels, they proposed exploring biogeochemical models. Doraiswamy illustrated the steps in these assessments and detailed the many components input in the EPIC-CENTURY model used to simulate carbon changes in soils with a two-year maize and soybean rotation, and under different tillage practices in Iowa. First, hyperspectral- and high-resolution imagery defined management zones, stratified sampling of soil measured the carbon content, biogeochemical modeling (integrating remote sensing parameters) scaled carbon sequestration, and finally, potential spatial and temporal sequestration was mapped. The models were applied for 25 years from 1970 to 1994 under conventional tillage, and then for another 25 years (1996-2020) with conventional tillage, mulch tillage, no-till, and no-till with rye crop. The early years incorporated recorded data on fertilizer application, climate, the soils' chemical and physical properties; these were included in the later years with rates of fertilizer applications adjusted after 2002 to maintain yields at the 2002 level. The EPIC model yielded valuable data, capturing most of the complex biogeochemical process in agriculture; compared to the earlier 25 years, simulation of soils in 2020 revealed a drop in carbon content in conventionally tilled soils with maize/soybean rotation, mulch tillage sequestered some carbon above the estimated baseline loss, and no-till soils showed a good absolute gain. The findings were shown in several maps from $25 \mathrm{~km}$ down to $30 \mathrm{~m}$ resolutions, including a map of $\%$ organic matter.

Doraiswamy drew attention to the pressing problem of erosion in soils under normal tillage that lowers the rate of carbon sequestration. Erosion is closely related to the particular landscape. Their researches, in two 1 x 1 mile plots, showed a loss of $2 \mathrm{~cm} /$ year on a $2 \%$ slope. Their recent work assessing the effects of the level of crop residues, using mid-infrared techniques, demonstrated their value in reducing erosion and conserving carbon stocks; here, management practices are important, especially in sloping landscapes. A short discussion followed about whether soil lost by erosion at elevated sites is accumulated at lower ones, so there is no overall loss of carbon. Presently, such information is lacking.

Allen Torbert, USDA, next considered Cost Assessment of Standard Soil Sampling. His objectives in this study were to see whether it was possible to detect small changes due to management and the environment against the inherent spatial and depth variability in soils. These needs, centered on measuring sequestration, differ from those of traditional soil sampling. Soils from different depths cannot be homogenized, since knowing the changes in the top 5$10 \mathrm{~cm}$ is important in accounting for carbon sequestration at least in the short-term, and it is 
critical to employ methods able to detect small changes. He illustrated how drastic differences in management can affect the soil's carbon levels, depending upon the type of soil. Torbert contrasted the practice of taking samples for traditional agronomic purposes with that for precision purposes, the former involving 10-35 samples from each designated location in uniform fields, and the latter encompassing up to 45 non-random composite samples on an established sampling grid. Traditional sampling requires minimal equipment (a sample probe, a bucket, and a sample box) with almost negligible costs, which are absorbed by the farmer. Automated soil sampling that goes deeper and maintains the soil's increments might cost at a minimum about $\$ 4.90$ per sample. Moving to the costs of laboratory analysis, Torbert reported his findings from a survey of laboratories throughout the country, looking in particular at the comparatively inexpensive Walkley and Black wet chemical procedure (not very accurate for carbon), and the loss on ignition (LIO) method (accurate, but measures weight loss so requiring a regression analysis using selected soils for comparison), and the more expensive, but more precise total elemental analysis with additional steps in processing and combustion at $1000^{\circ} \mathrm{C}$. The first two are sufficiently accurate for routine soil analysis; they cost $\$ 3.73$ to $\$ 16.00$ per sample, with most $\sim \$ 5.00$. Total elemental analysis costs $\$ 3.75$ to $\$ 22.00$, with most samples $\sim \$ 10.00$. He then applied these figures to calculate the expense of making a traditional assessment of carbon in a 700 hectare area, including time, supplies, equipment, and the costs of laboratory analyses. For the 210 samples taken and analyzed the total amounted to $\$ 1,646.40$, or $\$ 2.35 / \mathrm{ha}$. In responding to questions about costs versus credits for sequestration, he allowed that fewer samples might suffice, but nevertheless, a great many still would be needed.

John Kimble presented the final paper in the session, Considerations and Procedures for Temporal Sampling of Soil Carbon (co-authored by Ronald Follett). Kimble drew attention to the extent of work going on with over 2 million samples collected yearly for assessing soil fertility. He considered the parameters of temporal sampling, i.e., whether a change was occurring, how deep, its cause, and expected rate. Then he discussed the properties of soil, viz., organic-, inorganic-, or stabilized carbon, the different fractions, and their depth, texture and mineralogy, in relation to the twelve major taxonomic Soil Orders. The following two maps, global soil Orders and global soil organic carbon (SOC) showed how the former affects the latter, as it also affects soil inorganic carbon (SIC) globally. He discussed, and showed examples of, three very different soil types, Spodosols, Mollisols, and Vertisols, and how their characteristics affect the depth profile of organic and inorganic carbon, and hence, sampling requirements. He also discussed soils in general, noting that when someone says they have a loam soil it may only reflect its surface texture and the soil's profile may be very variable with depth, i.e., loam over sand over clay, or any other combination. This can lead to serious complications in interpreting data unless this variation with depth is considered. Spodosols are acid soils, with an amorphous mix of organic matter complexed with aluminum, and sometimes, iron; these naturally infertile soils often occur in northern coniferous forests. Mollisols, typically in grasslands in middle latitudes, have a thick dark horizon high in organic matter and are very productive. The clay-rich Vertisols, many of which are in Texas, shrink and swell with moisture, thereby posing serious engineering problems; their cracking also results in deep mixing of fresh surface carbon into the soil's profile. Sampling in all of them requires preplanned protocols, and the establishment of microsites; otherwise, paired sampling might be suitable. He enumerated other temporal 
information needed for sampling, including GIS coordinates for the samples' locations, baseline measures and units of measurement, moisture content, and the history of the soil's treatments and management. He considered systematic and pre-planned protocols, and replicated sampling with time, suggesting looking at publications where these factors were detailed. He mentioned the advantages of considering golf courses to derive meaningful data (non-till) since excellent records are kept of the turf's treatment; his accompanying slide of three courses in Fort Collins, and three in Denver plotted total organic carbon in the native grassland 45 years before the courses were established, and demonstrated its increase thereafter throughout the following century. He showed how the data obtained from detailed samplings can be useful for modeling of such information.

Kimble explained the value of using the Paired Plot technique when only a single sample can be taken at each location, but suggested that paired plots could be treated as replications. It is also a useful approach when land ownership or management may change, and when comparing long-term and large-scale management systems. He briefly mentioned the isotopic method for studying temporal changes in soil carbon, measuring the ratio of ${ }^{13} \mathrm{C}$ and ${ }^{12} \mathrm{C}$ isotopes resulting from changes in vegetation, and incorporating the naturally abundant ${ }^{14} \mathrm{C}$ to assess paleoclimatic and vegetative changes. In conclusion, he re-emphasized the need to be cognizant of the soils' properties, depth, rates and causes of changes in carbon, and then to design the best feasible experimental design. Further, the scale of sampling and variability must be considered for carbon trading. Trading will be over very large areas and compost sampling may work the best for such estimates. Researchers should not focus on short-range variability all the time, but look at landscape units and apply our knowledge of soil science for making large-scale estimates of soil carbon. 


\section{Round Table Discussions and Recommendations}

Before beginning the discussions, the workshop's Organizer, Lucien Wielopolski, handed out a list of suggestions as the basis for consideration; there were three categories, Instrumentation, Requirements, and Recommendations, each with 4 or 5 items*. He hoped that the first two might focus the deliberations, and lead to important, cohesive, and collaborative paths forward in the latter.

Session 1. Characteristics and Merits of the New Technologies versus the "Gold Standard Moderator: Rattan Lal, Ohio State University

Lal opened the discussion by posing a question, the answer to which he believed would be important: What do scientists need to do to monitor carbon in a new way? He started with a brief look at the history of soil carbon assessments, starting with the Spanish Moors in the $12^{\text {th }}$ century A.D. to the first published paper in Germany in 1860, through to the Soil Organic Management IUSS in New Delhi in 1982. These early documents mentioned several vital parameters, viz., relating changes in the quality and color of the soil to its carbon content, and discussed the effects of management. Indeed, in his chapter in "Soils and Men", 1938, Daniel Albright deemed soil organic matter the nation's most precious resource. Over the years, the focus has been on the soils' fertility, and maintaining its quality, and hence, productivity. Lal contended that there is a big disconnection in soil science between this history of research focused on fertility, and future directions in which research must move to measure carbon sequestration. Agreeing with Roger Dahlman's opening comments, the effectiveness of emerging technologies cannot be predicted because the fundamental chemical, physical, and biological processes that underlie carbon sequestration, and vice versa, are incompletely understood, nor are there sufficiently good predictive models.

In the ensuing discussion, the opinions of participants were divided about this; some agreed that soil scientists must be challenged to radically alter their way of thinking and approach, and that the fundamental questions have changed in moving from enhancing fertility and crop production to considering carbon mass in the soil as a repository for 30 to 40 years. One participant thought that investigations were "stuck in a mode". However, others thought that already there is more than a sound basic knowledge of the processes taking place, their complexity, and their rates, upon which to build. Thus, although historically sequestration was not considered in surveys, analyses, and experiments, the USDA's new program GRACEnet (Greenhouse gas Reduction through Agricultural Carbon Enhancement network) is generating robust information from it; further, people in other disciplines, such as microaggregate research, know much that is relevant. Researchers know the basic processes that must be measured. Another participant repudiated the view that previous researchers were interested only in the top $30 \mathrm{~cm}$ of soil.

Despite these different opinions about the value of earlier work to present-day concerns, there was general agreement that attention must turn to developing methods to assess changes over large areas over longer times, i.e., scaling, and on accounting for the soil's variability. The scope of the knowledge of processes gained site by site must be integrated and extended both spatially and in depth - the role of ancillary processes and drivers must be explored. Again, 
participants concurred in expecting that the new instrumentation, along with meaningful simulations and the attendant calculations would usher in major insights into the processes controlling carbon uptake in soils. The comment was made that after 200 years of measurements, shovels still were being used! The needs for monitoring, and assessments at landscape levels might be resolved to some extent, provided that the instruments are developed quickly, and while the resulting picture might lack finer details, a rough draft might be very advantageous. A hierarchy within scientific research was proposed as an interim measure, with layers of researchers undertaking field experiments in coordination with others working at landscape levels and upward.

Lal turned next to the progress that had been made in instrumentation, and whether these advances would address the pressing research needs. His first slide set out the unique characteristics of the INS system - its non-destructiveness, in situ assessments, and ability to scan large volumes and areas. His second one listed the advantages of LIBS; its portability, the small volumes needed, and its sensitivity (LOD better than $0.1 \%$ carbon). He compared these, and the infrared spectroscopic instruments, with the crude "Gold Standard" methodology that lacks sensitivity, introduces variability, and is costly.

The following discussion touched upon several questions: How do roots and rocks in the soil affect scattering? What volume is being measured? How do the findings relate to assigning carbon credits and trading? General agreement was that rocks in soil present a real problem; their presence is apparent in scans from both INS and LIBS, and if infrared techniques can distinguish between inorganic and organic materials, computer programs might separate them. It was suggested simply accepting that roots are part of the total carbon in a sample, and scaling up accordingly to see how management practices alter carbon content. Regarding the measured volume, the radiation signal from the INS continues down with depth, so that results reflect the number of carbon atoms in that "infinite" volume, and can be expressed in relative terms. By doing so, calibrations would not be required. Relative changes also might be important in affording credits regionally. In terms of carbon trading (or, properly, carbon dioxide credits), integrating the findings from instruments with those from laboratory analyses might be satisfactory; several participants opined that no one methodology alone would provide answers. Again, rather than focusing on technological advances and accuracy, researchers might concentrate on improving sampling and establishing protocols for expressing relative changes at landscape levels; in other words, rather than seeking improvements, use the methodologies available better. One attendee strongly favored the power afforded by involving statisticians in the work, not only in establishing sampling procedures but in examining the accuracy of instruments and in cross validations. Indeed, the great statistician, Ronald Fisher, did much of his innovative work at the Rothamsted Experimental Station elaborating methods to deal with reallife variability in agriculture.

Lal discussed the problems of calibrating instruments and methodologies. He asked at which point huge roots, tropical grasses, and large mats of roots are sequestered to become part of the soil's carbon stores, and whether different calibrations are required for different soil types and textures, i.e., clay, silt, and sand. One of his accompanying slides, from samples taken in Duke Forest, showed the mean volume of coarse aggregates ranging from 0 to $48 \%$ (mean $12 \%$ ). Carbonates in the soil also posed a problem for calibration. The opinions expressed by the audience were many and various. All agreed that often the soils' profiles were stratified 
vertically and horizontally; some suggested that calibration could be avoided by sampling at time zero and at intervals later, although the problems of extrapolating from local to regional levels remained. Evaporating the samples might be a useful technique since it eliminates matrix effects. Others were in favor of having separate calibrations for soil types. However, this might entail having an enormous library for soils globally, with up to 400,000 samples, yet it was hard to see the day when research would be calibration-free. Several considered limiting the number of calibrations by merging and combining them by region, so that they would be applicable to, and properly representative of, millions of acres. One suggestion was that separate calibrations might be needed for low and high levels of carbon. Lal concluded that the calibration of instruments, as with other methods, would have to be based on supplementary data from analyses of samples, and from reference materials.

Lal led the last discussion of his session that first considered problems of carbon erosion/deposition, and then touched upon the overall role of the new instruments. Thus, how can the dynamics of the erosion and deposition of carbon be expressed, bearing in mind, for example, that the erosions and movements of soil from the top to the bottom of a hill does not directly represent its sequestration there. Further, erosion breaks down aggregates so that carbon becomes more labile, and up to $20 \%$ may then be lost to the atmosphere. A landscape model of erosion is required. The INS can scan uphill and downhill and obtain assessments. The value was debated of measuring surrogates for carbon, such as minerals and even toxic materials, and using infrared techniques qualitatively, rather than quantitatively, to discern things such as highmolecular lipids bound to carbon. Similarly, depth-related chemical assays have revealed that such lipids are specific to one type of soil. Models of carbon movement were said to have inadequate spatial sensitivity - soil scientists might turn to the research on water quality for ideas. In closing the discussion, Wielopolski saw an important role for instruments as together they offer a multidimensional answer, and the validity of the findings can be demonstrated. He expressed concern that there was no clear consensus from the discussion on what they can particularly offer.

Session 2. Recommendations for New Calibration and Sampling Procedures and Identification of Reference Sites and Synthetic Soils.

Moderator: Roger Dahlman, DOE.

Dahlman moved the core of the discussion to listing critical questions on which the science might concentrate, as suggested by Wielopolski. These would include the requirements for measurements, and the effectiveness of the emerging technologies in assessing carbon sequestration. The first one offered was to construct an uncertainty budget in enumerating the contribution of, and sources of, error propagation at each step in a process. The importance of this idea was recognized and it was accepted enthusiastically. Mention was made of charting comparative coefficients of variation at each step, and of the value of adding error bars on measured values. One contributor asked what would be acceptable limits on the error term and how this might relate to carbon credits. One respondent believed that acceptable error would be dictated by the price of carbon, or in other terms, risk to the company buying carbon credits would determine the allowable error; then, could the concept of error acceptance for the carbon commodity be added to the critical list? The general feeling was that this was outside the 
purveyance of soil scientists and rests with private enterprise, and should not be included in the list of critical issues. The question was raised about the costs of reducing error, compared with those of taking more samples; perhaps, instead, efforts should focus on increasing the accuracy of methods and instruments, ensuring they are deployable in the field, and leaving the policymakers to state the needed accuracy.

The next debate centered on testing field-deployable instruments, singly or in concert, and preferably in the field. The first discussant suggested this avoided answering what were the general requirements of them. Under the Kyoto Protocol, credits are given for $0.1 \%$ annual sequestration, or 1ton change/hectare/field, that probably cannot yet be measured, though probably such a change could be detected in 3 to 5 years. Hence, tests should be geared towards assessing the instrument's ability to do this. In turn, testing may require high-precision instruments for validation. Already, the INS system working in environments where carbon levels in the soil are $1-2 \%$ can register a change of $0.05 \%$. Another participant commented that a yearly $1.1 \%$ increase in carbon can be detected over three years at the landscape level.

The meeting explored the value of having reference materials for calibrations. So far, there are very few for carbon or for partitioning it, although standards exist for carbonate. The U.S. Geological Service has new soils and SOMD (Soil Organic Matter Dynamic) soils from Nebraska but they may require certification. However, costly suites of standards, including extremes, may be needed to test all the instruments. Standard reference materials are needed, and their formulation should be taken into account. That is, should they be synthetic materials, or should real soils be fortified with the compound of interest? The suggestion was made to develop "lab dirt" the parameters of which would be easily changed. Cautions were expressed about the effect of loss of the soil's matrix structure and differences in porosity in the reference samples, so real soils may be preferable. Since there is a dearth of reference materials, the suggestion was made that several well-characterized benchmark sites might be established for testing and optimizing the range of new instruments. But since these sites are dynamically changing, a further suggestion was to set up pits containing different well-characterized soils in large sheds, an approach toward attaining the stability of laboratory conditions, and to use standardized test protocols. These pits might contain graphite rods to see where the measurement was being obtained. Several participants volunteered to explore all aspects of this idea further, and propose sites and specifications. Funding through the Soil Science Society of America might be explored.

The group then debated optimizing the sampling protocols, considering collection depth, number of samples, and, for instruments, the area to scan. The importance of knowing the bulk density of the soil was reiterated. There were some questions on whether attention should be paid first to establishing protocols then testing instruments, or vice versa. In view of the need for high-quality measurement technologies, participants favored the latter approach. Optimizing the instruments will encourage their wider use and evaluation, so that some modalities might be combined. The question about standard soils came up again in the context of validating the instruments that is essential when measuring such small increases in carbon. This may involve having synthetic soils, and natural ones both with and without additives (including root, leaves, and rocks, as well as chemicals). One participant felt that this detailed discussion was moving away from listing the critical areas for research. The debate seemly showed that more than one instrument would be required to meet all needs; indeed, in rocky soils, shoveling may be a prerequisite. Different calibrations may be required for the same instrument working on different 
soils, and further, the methodology must be tied to the specific objectives. In all this work, complementary approaches must be employed, including the "gold standard" because deploying the instruments worldwide would be too costly. It was suggested that a statistician might be prevailed up to evaluate and compare the costs of the many factors involved, including labor, preparation, the required coverage, and the expenses of the instruments, simple or complex.

The session closed with a short discussion of choosing location of the standard experimental sites, a proposal that participants agreed upon, and where to seek funding for this endeavor. Since government funding may not cover testing of the new instruments, other sources will have to be sought. 


\section{SUMMARY}

Workshop Summary from the Organizer's Point of View:

Predictions of worldwide energy use in the next century suggest a continued increase in carbon dioxide $\left(\mathrm{CO}_{2}\right)$ emissions into the atmosphere that will, in turn, accelerate global warming. Several approaches to mitigate this scenario are being considered on the production and on the user side. One promising approach to reducing atmospheric $\mathrm{CO}_{2}$, namely, by increasing its sequestration, stands out as a potent and viable way of arresting, if not stopping, the buildup of $\mathrm{CO}_{2}$. Of the several ways in which carbon can be sequestered, that within the terrestrial ecosystem can best be manipulated to our advantage, since, in essence, it serves as a huge natural biological scrubber for $\mathrm{CO}_{2}$. Several of the DOE's Terrestrial Carbon Processes (TCP) road-map outlines and mission statements on carbon sequestration that pertain to the long-term continentalscale analysis and prediction of the carbon cycle by the North American Carbon Plan, emphasize the need for better understanding its belowground processes, and transfer rates, together with a better assessment of the soil's carbon stocks. Cardinal to meeting all these goals is having novel and improved soil-carbon measurements. Presently, soil carbon is assessed directly by taking samples to a laboratory for dry-combustion chemical analysis, and indirectly by eddy covariance flux measurements. Although these two methods afford basic information on the carbon levels in soil, they are inadequate for meeting the demands of the ever-growing number of sites that must be sampled, and for delivering more precise, detailed information on carbon distribution.

A new generation of analytical instrumentation for analyzing soil composition was introduced in a special workshop, the first of its kind dedicated to novel approaches for carbon analysis. The workshop explored several new in situ technologies that are non-destructive, inexpensive, and less labor-intensive than common present-day methods. Furthermore, they operate in static and dynamic modes, and can provide more detailed information on the chemical- and elemental-speciation of the belowground carbon, features currently not available. The basic premise of the workshop was that no single modality can resolve all the issues; thus, operating them in tandem might provide the ideal situation for studying the belowground processes. For such system, integration and proper interpretation of data it is highly desirable to understand a) what each modality is measuring, b) how is it being measured, and, c) what is the volume that is being sensed by the detection system.

The four different modalities presented for carbon analysis included 1) Laser Induced Breakdown Spectroscopy (LIBS), wherein a soil volume of few millimeters cubed from an extracted core is vaporized into plasma, and an optical spectrum is acquired from the cooling ions, 2) Mid- and Near- Infrared Spectroscopy (MIR and NIR) in which 0.5- to 3-centimeters cubed of soil from a sample is irradiated with infrared radiation which stimulates the molecular vibrations as it diffuses and eventually is reflected back. Peaks in the reflected spectrum correspond to the resonance absorptions in the soil, 3) Pyrolysis Molecular Beam Mass Spectroscopy (Py-MBMS) in which small, $\sim 0.5 \mathrm{~g}$, soil samples are introduced to a molecular beam mass analyzer that records the masses of all the species in the sample. The system is sensitive to chemical speciation of the molecules analyzed, and employs data analysis for sorting the multiplicity of spectral lines, and, 4) Neutron Inelastic Scattering (INS) that involves irradiating the soil with fast neutrons and measuring the induced gamma rays. This nuclear 
method is insensitive to the soil's chemical composition, and thus, it measures purely elemental carbon and other elements. Since it involves high-energy radiations, the soil volume analyzed is about 0.3 cubic meters. The INS system, which hovers about $30 \mathrm{~cm}$ above the ground, is totally non-destructive and can be used in static or scanning modes.

Speakers at the Workshop introduced some details of the systems; Table 1 summarizes

\begin{tabular}{|c|c|c|c|c|c|c|}
\hline Instrument & Process & Methodology & \begin{tabular}{|c|} 
Radiation \\
Type \\
Detected
\end{tabular} & $\begin{array}{l}\text { Penetration } \\
\text { Depth }(\mathrm{cm})\end{array}$ & $\begin{array}{c}\text { Sampled } \\
\text { Volume } \\
\left(\mathrm{cm}^{3}\right) \\
\end{array}$ & $\begin{array}{c}\text { Measurement } \\
\text { Type }\end{array}$ \\
\hline LIBS & Atomic & $\begin{array}{c}\text { Plasma } \\
\text { Induced } \\
\text { Emission } \\
\end{array}$ & Visible & $\sim 0.1$ & $10^{-2}$ & \begin{tabular}{|c|} 
Point, \\
Depth Profile, \\
Destructive \\
\end{tabular} \\
\hline NIR & Molecular & $\begin{array}{c}\text { Diffuse } \\
\text { Reflectance }\end{array}$ & $\begin{array}{l}\text { Near- } \\
\text { Infrared }\end{array}$ & $\sim 0.2$ & $10^{0}$ & $\begin{array}{c}\text { Surface, } \\
\text { Scanning, } \\
\text { Destructive }\end{array}$ \\
\hline MIR & Molecular & $\begin{array}{c}\text { Diffuse } \\
\text { Reflectance }\end{array}$ & Infrared & $\sim 1$ & $10^{1}$ & $\begin{array}{c}\text { Surface, } \\
\text { Destructive }\end{array}$ \\
\hline Py-MBMS & Molecular & $\begin{array}{c}\text { Pyrolysis } \\
\text { Molecular } \\
\text { Beam }\end{array}$ & $\mathbf{m} / \mathbf{z}$ & --- & $10^{1}$ & $\begin{array}{l}\text { Samples } \\
\text { Destructive }\end{array}$ \\
\hline INS & Nuclear & $\begin{array}{c}\text { Neutron } \\
\text { Induced } \\
\text { Nuclear } \\
\text { Reactions }\end{array}$ & $\begin{array}{c}\text { Gamma } \\
\text { Rays }\end{array}$ & $\sim 30$ & $10^{5}$ & $\begin{array}{c}\text { Volume, } \\
\text { Scanning, } \\
\text { Non- } \\
\text { Destructive }\end{array}$ \\
\hline
\end{tabular}

some of the more general physical characteristics. However, due to time limitations, speakers did not address all of the questions posed at the beginning of the workshop, so that many critical details important for thoroughly evaluating all the systems were not discussed. For example, the systems' interferences and their effects on spectral analysis strongly depend upon the type of measurement. All of the systems suffer either from elemental or matrix interferences, although not all the speakers brought this up. Details on the calibration procedures in some cases were missing. Many different quantities related to, associated with, or proportional to, carbon in its various forms, can be calibrated against the independent quantity of carbon content in soil, as determined by combustion chemistry, assuming for now that the latter is free of problems. Again, knowing precisely of what is being measured and knowing the corrections for the interferences may assist in establishing the systems' calibration and its dependence on soil type, composition, morphology, and other field variables. Similarly, not all of the systems offer the sensitivity, defined as the system's response, in system's units, per unit carbon in soil, which is the slope of the calibration line, nor do they provide the minimum detectable limit or change. Finally, it is pertinent that the measurement error is reported for each method; this value is essential for reducing error propagation for up-scaling, or for assessing confidence levels on the carbon estimates. Having all this information will assist in establishing the best calibration procedures 
for each system and optimizing its use. Some of the issues requiring clarifications for each system are discussed below.

LIBS- Several issues in the LIBS system remain unclear. The first pertains to the volume size that the laser pulse vaporizes (converts to plasma). It appears to depend on the matrix's composition, moisture content, and morphology, together with its porosity, and bulk density; the effects of these parameters on the signal's yield need to be evaluated separately. A second issue is the striking multiplicity of elemental and molecular spectral lines generated when the plasma cools that poses the question of what interferences and correction factors should be applied, if any, in data analysis. Clarifications on spectral analysis would be helpful. A third issue centers on the detection limits and how they are defined. Since LIBS uses a narrow beam, which penetrates randomly into the porous core, probably there will be good shots, poor shots, and misses; therefore, the detection limit cannot be defined as the mean value of the detected signal because it would depend on the soil's overall morphology. Hence, what is meant when speakers report detection limits? Addressing these questions and explaining the plasma-cooling process would be very helpful to further the use of LIBS.

MIR- and NIR- The responses to matrix effects of the two infrared systems operating at two different wavelengths, MIR $\sim 6000 \mathrm{~nm}$ and NIR $\sim 1500 \mathrm{~nm}$, are not identical. Nonlinear calibration suggests that the signal is not proportional to carbon content and that chemical bonds interfere with carbon determination. The magnitude of these matrix effects on signal intensity and the sampled volume are not well defined in these methods; systematic studies of the effects of moisture and bulk density also would be very helpful. Similarly, spectral analysis and data interpretation is not straightforward and clarifications of the characteristics of its PCA (Principal Component Analysis) software would be useful. The errors involved with the infrared systems were not reported.

Py-MBMS- This is more standardized use of an established analytical methodology. Clarification is needed how a pyrolytic reactor is controlled to prevent breakdown of the compounds used for various identifications in SOM. The multiplicity of spectral lines probably suffers from interferences, and thus, how the PCA software handles the analysis and error propagation.

INS- This is a nuclear method totally insensitive to chemistry of the carbon; thus, it measures total carbon. Because of the high energies of the neutron- and gamma-radiations it measures large volumes of about $0.3 \mathrm{~m}$ cube, as assessed from an analytical simulation of the system. However, like with the other systems the precise volume sampled remains to be determined experimentally or at least it must be demonstrated that this large volume is affected very little by soil parameters, such as density and moisture.

None of these systems is finalized and attempts were made to point out where major effort is required to bring them to completion. Nevertheless, the systems are mature enough to bring them together for field evaluation, and see how they complement each other.

The scarcity of soil standards for in situ systems calibration was recognized and no resolution is in sight; one limiting factor was the amounts that would be required. Instead, the 
feasibility of using standardized fields or synthetic soils was raised. Finally, before the systems are completed and fully characterized and understood it is difficult to design or recommend a sampling paradigm for each system. This will depend on nature of the measurement, and the volumes involved that, in turn, affect the measurement errors. In short, new technologies are coming that soon might replace the old faithful shovel. However, before this happens all the systems should be brought side- by-side to well controlled experiments in fields with various landscapes, soil structure and content, to identify the limitations of each system in analyzing rocky, forest, and wet terrains. 


\section{APPENDIX A:}

\section{Agenda}

January 18, 2006 (Wednesday), Brookhaven Center

17:00 - 20:00 Pre-workshop Social

January 19, 2006 (Thursday), Large Conference Room Bldg. 490

\begin{tabular}{|c|c|c|}
\hline $8: 00-9: 00$ & Continental Breakfast & \\
\hline $\begin{array}{l}9: 00-9: 10 \\
9: 10-9: 15 \\
9: 15-9: 25 \\
9: 25-9: 35 \\
9: 35-10: 00\end{array}$ & $\begin{array}{l}\text { Welcome } \\
\text { Welcome } \\
\text { Introduction } \\
\text { Workshop Objectives } \\
\text { Coffee Break }\end{array}$ & $\begin{array}{l}\text { Creighton Wirick, Chair } \\
\text { Ralph James, Ass. Lab. Dir. } \\
\text { Roger Dahlman, DOE } \\
\text { Lucian Wielopolski, BNL }\end{array}$ \\
\hline Instrumentation & Session Chair, & John Litynski, DOE/NETL \\
\hline 10:00 - 10:20 & $\begin{array}{l}\text { Laser Induced Breakdown } \\
\text { Spectroscopy (LIBS) }\end{array}$ & Ronny Harris, LANL \\
\hline 10:20 - 10:40 & $\begin{array}{l}\text { Near Infra-Red Spectroscopy } \\
\text { (NIR) }\end{array}$ & Colin Christy, VT \\
\hline $10: 40-11: 00$ & $\begin{array}{l}\text { Mid Infra-Red Spectroscopy } \\
\text { (MIDR) }\end{array}$ & Jim Reeves, USDA \\
\hline $11: 00-11: 20$ & $\begin{array}{l}\text { Pyrolysis Molecular Beam Mass } \\
\text { Spectrometry (py-MBMS) }\end{array}$ & Kimberly Magrini, NREL \\
\hline $11: 20$ - 11:40 & $\begin{array}{l}\text { Inelastic Neutron Scattering } \\
\text { (INS) }\end{array}$ & Lucian Wielopolski, BNL \\
\hline $\begin{array}{l}11: 40-12: 00 \\
12: 00-13: 00\end{array}$ & $\begin{array}{l}\text { Gold Standard - The Common } \\
\text { Approach for Measuring Soil C } \\
\text { Amounts } \\
\text { Working Lunch }\end{array}$ & Achim Dobermann, UNL \\
\hline Current Soil Sam & 1 Practices Session Chair, & Nancy Cavallaro, USDA \\
\hline $\begin{array}{l}13: 00-13: 30 \\
13: 30-14: 00 \\
14: 00-14: 20\end{array}$ & $\begin{array}{l}\text { Spatial Sampling Methodologies } \\
\text { and Practices: Bulk Density } \\
\text { Monitoring Spatial and Temporal } \\
\text { Carbon Changes in the Field } \\
\text { Strategies for Monitoring and } \\
\text { Mapping of Soil C in Large } \\
\text { Fields }\end{array}$ & $\begin{array}{l}\text { Cesar Izaurralde, PNNL } \\
\text { Chuck Rice, KSU }\end{array}$ \\
\hline
\end{tabular}




\begin{tabular}{|c|c|c|}
\hline $14: 20$ - 14:40 & Unique Aspects of Sampling & Kurt Johnsen, USDA \\
\hline 14:40 - 15:00 & $\begin{array}{l}\text { Sampling Procedures, Sampling } \\
\text { Volume and Accuracy }\end{array}$ & Harold Van Es, Cornell U. \\
\hline $\begin{array}{l}\text { 15:00 - 15:10 } \\
\text { New Standards } \\
\end{array}$ & $\begin{array}{l}\text { Coffee Break } \\
\text { Session Chairman }\end{array}$ & Tristram West, ORNL \\
\hline $15: 10-15: 30$ & $\begin{array}{l}\text { Scanning Versus Static Soil } \\
\text { Sampling }\end{array}$ & Lucian Wielopolski, BNL \\
\hline $15: 30-15: 50$ & $\begin{array}{l}\text { Calibration, Validation and the } \\
\text { Question of Outliers }\end{array}$ & David Brown, MSU \\
\hline $15: 50-16: 10$ & $\begin{array}{l}\text { Reference Materials and } \\
\text { Synthetic Soils }\end{array}$ & Ben Ellert, AGR Canada \\
\hline $16: 10$ - 16:30 & GIS and Up-Scaling & Paul Doraiswamy, USDA \\
\hline $16: 30-16: 50$ & $\begin{array}{l}\text { Costs Assessment of Standard } \\
\text { Soil Sampling }\end{array}$ & Allen Torbert, USDA \\
\hline $16: 50-17: 10$ & Temporal Soil Carbon Sampling & John Kimbel, USDA \\
\hline 19:00 & & \\
\hline
\end{tabular}

January 20, 2006 (Friday), Large Conference Room Bldg. 490

8:00 - 9:00 Continental Breakfast

$\underline{\text { Round Table Discussion and Recommendations }}$ Moderators

\begin{tabular}{|c|c|c|}
\hline $9: 00$ - 11:00 & $\begin{array}{l}\text { Characteristic and Merits of the } \\
\text { New Technologies versus the } \\
\text { "Gold Standard" }\end{array}$ & Rattan Lal OSU \\
\hline $11: 00-13: 00$ & $\begin{array}{l}\text { Recommendations for New } \\
\text { Calibration and Sampling } \\
\text { Procedures and Reference Sites } \\
\text { Versus Svnthetic Soils }\end{array}$ & Roger Dahlman, DOE \\
\hline
\end{tabular}




\title{
APPENDIX B
}

\author{
Abstracts \\ Analysis of Total Soil Carbon with Laser-induced Breakdown Spectroscopy (LIBS)
Ronny D. Harris and Michael H. Ebinger, Earth and Environmental Science Division (EES-2), Los Alamos National Laboratory, Los Alamos, NM 87545

\section{Introduction}

Loss of soil carbon during the past 150 years has depleted many of the most productive soils and resulted in degradation of marginal soils upon which many people depend. The potential to restore carbon stocks in different landscapes through careful land management practices could result in significant improvements to crop production in agricultural lands and overall soil quality improvements in other lands (Lal, 2004; IPCC, 2000). Increases in soil organic carbon (SOC) depend on climate and management practice, and potential increases are estimated from 0 to $150 \mathrm{~kg}-\mathrm{C}$ ha-1 $\mathrm{yr}-1$ in semiarid environments up to $1000 \mathrm{~kg}-\mathrm{C}$ ha- $1 \mathrm{yr}-1$ in more humid environments (Lal, 2004; Armstrong et al, 2003; West and Post, 2002). Current methods of carbon analysis (e.g., Rossel et al., 2001; Scharpenseel et al., 2001) provide the analytical tools needed to estimate these increases in the SOC pool with some precision.

Advanced analytical methods (e.g., Ebinger et al., 2003; McCarty et al., 2002; McCarty and Reeves, 2001; Cremers et al, 2001) offer improved detection, ease of operation, and potential use in the field that could improve precision and accuracy of SOC measurements. In addition, the need for improved accuracy and precision to support national and international policies on carbon emissions and carbon trading may require orders of magnitude more measurements to provide valid support for various positions and land management practices. These measurements must be delivered at the lowest cost possible and with well-characterized uncertainties. Current methods fall short on cost effectiveness as well as accuracy and precision; advanced methods, once fully developed and tested, should optimize the amount of information about SOC pools per dollar spent, and must be designed to keep the cost of assessing carbon to less than $10 \%$ of the total costs of sequestration practices (DOE, 2004). With sequestered carbon becoming a commodity in the United States and Europe, the need to measure and certify increases (or decreases) in SOC within 3 to 5 years of implementing carbon sequestration and management practices, and to do so at the lowest levels of detection, is a pressing issue.

Developing more cost effective and rapid methods of measuring soil carbon is important to address aspects of global climate change and terrestrial carbon management issues. Over the past two decades, several advanced analytical methods have been applied to the study of soil carbon. Laser-induced breakdown spectroscopy (LIBS) is a technique similar to atomic emission spectroscopy (AES) that can be developed for soil carbon analysis.

\section{LIBS Method}

A laser is focused on a solid sample and forms a microplasma as the sample is ionized. The light from the microplasma is characteristic of the elemental composition of the sample. This emitted 
light is collected, spectrally resolved, and detected to monitor concentrations of elements via their unique spectral signatures. When calibrated, the method provides quantitative measurements; when applied to soils, carbon and most other elements present in soil samples can be quantified in seconds. Strong carbon C(I) emission lines at $247.8 \mathrm{~nm}$ or $193 \mathrm{~nm}$ are used to create calibration curves and quantify soil carbon (Ebinger et al., 2003; Cremers et al., 2001). Approximately 100 laser shots are directed onto a sample to complete one measurement. Typical measurement areas for the LIBS analysis are 1 to $5 \mathrm{~mm} 3 / \mathrm{pulse}$, and collection of one measurement takes about 10 seconds. Peak heights or areas from $247 \mathrm{~nm}$ or $193 \mathrm{~nm}$ are recorded for the carbon emission line chosen, and background signals from the spectra are subtracted; this procedure is repeated for each soil sample.

\section{Analysis of Discrete Samples}

LIBS has been tested for carbon analysis by using soils collected during soil surveys in the United States. Soils of differing texture, mineralogy, and several other soil properties as well as carbon concentration have been analyzed. Soil samples are pressed into $30 \mathrm{~mm}$ discs before completing a carbon measurement. The LIBS signal intensity was found to be a function of soil carbon concentration. Instrument calibration is achieved by comparing the LIBS signal to dry combustion analysis. Limits of detection are better than $0.1 \%$ carbon.

\section{Analysis of Intact Cores}

A LIBS instrument has been modified to analyze intact cores of soil extracted with a soil probe (up to $1.3 \mathrm{~m}$ in length and $4 \mathrm{~cm}$ diameter). The primary goal of instrument development was to create a new analysis method that could be used in the field with little or no sample preparation. After extraction of the core, a window is cut in the plastic tube containing the soil to allow access by the laser. The core is then placed on the track of the LIBS instrument and 10 to 100 shots are collected at $1 \mathrm{~mm}$ intervals along the length of the core. These scans provide unique highresolution distribution of carbon concentration in the extracted core, a feature that cannot be provided using conventional analysis. Instrument calibration is also achieved by comparing the LIBS signal to dry combustion analysis.

\section{Conclusions}

LIBS instruments are field portable and require little to no additional preparation of the soil samples. Soil carbon can be analyzed quickly, accurately, and precisely with LIBS while saving time and money when compared to conventional methods (dry combustion). LIBS analysis of intact cores is a promising new method providing high resolution carbon distribution data (depth profiling of total carbon) that is not possible with other methods.

\section{References}

Armstrong, R. D., G. Millar, N. V. Halpin, D. J. Reid, and J. Standley. 2003. Using Zero Tillage, Fertilisers, and Legume Rotations to Maintain Productivity and Soil Fertility in

Opportunity Cropping Systems on Shallow Vertisol. Aust. J. Exp. Agriculture 43:141-153. 
Cremers, D. A., M. H. Ebinger, D. D. Breshears, P. J. Unkefer, S. A. Kammerdiener, M. J. Ferris, K. M. Catlett, and J. R. Brown. 2001. Measuring Total Soil Carbon With Laser-Induced Breakdown Spectroscopy (LIBS). J. Environmental Qual. 30:2202-2206.

Department of Energy (DOE). 2004. Carbon Sequestration Technology Roadmap and Program Plan-2004. National Energy Technology Laboratory, DOE Office of Fossil Energy (available at: http://www.netl.doe.gov, accessed September 1, 2004).

Ebinger, M. H., M. Lee Norfleet, D. D. Breshears, D. A. Cremers, M. J. Ferris, P. J. Unkefer, M. S. Lamb, K. L. Goddard, and C. W. Meyer. 2003. Extending the Applicability of Laser-Induced Breakdown Spectroscopy for Total Soil Carbon Measurement. Soil Sci. Soc. Am. J. 67:16161619.

Intergovernmental Panel on climate Change (IPCC), 2000. Land Use, Land Use Change, and Forestry. Cambridge Univ. Press.

Isaacs, E. H. and R. M. Srivastava. 1989. An Introduction to Applied Geostatistics.

Lal, R. 2004. Soil Carbon Sequestration Impacts on Global climate Change and Food Security.

Science 304:1623-1627.

McCarty, G.W., and J.B. Reeves III. 2001. Development of rapid instrumental methods for measuring soil organic carbon. p. 371-380. In R. Lal et al. (ed.) Assessment methods for soil carbon. Lewis Publ., Boca Raton, FL.

McCarty, G.W., J.B. Reeves III, V. B. Reeves, R. F. Follett, and J. M. Kimble. 2002. MidInfrared and Near-Infrared Diffuse Reflectance Spectroscopy for Soil Carbon Measurement. Soil Sci. Soc. Am. J. 66:640-646.

Rossell, R.A., J.C. Gasparoni, and J.A. Galantini. 2001. Soil organic matter evaluation. p. 311322. In R. Lal et al. (ed.) Assessment methods for soil carbon. Lewis Publ., Boca Raton, FL. Scharpenseel, H.W., E.M. Pfeiffer, and P. Becker-Heidmann. 2001. EcozonRossell, R.A., J.C. Gasparoni, and J.A. Galantini. 2001. Soil organic matter evaluation. p. 311-322. In R. Lal et al. (ed.) Assessment methods for soil carbon. Lewis Publ., Boca Raton, FL.

West, T. O. and W. M. Post. 2002. Soil Organic Carbon Sequestration Rates by Tillage and Crop Rotation. Soil Sci. Soc. Am. J. 66:1930. 


\section{In-situ Measurement of Soil Carbon by Near Infrared Diffuse Reflectance Spectroscopy Colin Christy, Ph.D. Veris Technologies}

Near infrared (NIR) diffuse reflectance spectroscopy measuring the diffusely scattered light from an illuminated sample, which, in general, the light has penetrated and induced molecular vibrations. The vibrations absorb a portion of the light energy, so that a mathematical comparison of the spectra from the incident- and reflected-light can indicate the type and quantity of certain molecules in the sample.

Reflectance in the near infrared portion of the electromagnetic spectrum is highly influenced by molecules containing strong bonds between relatively light atoms. These bonds tend to absorb energy at overtones and combinations of the fundamental vibrational frequencies of the mid infrared. The predominant absorbers in the near infrared region are the $\mathrm{C}-\mathrm{H}, \mathrm{N}-\mathrm{H}$, and $\mathrm{O}-\mathrm{H}$ functional groups, making this region ideal for quantifying forms of carbon, nitrogen, and water, respectively. Values are obtained using chemometric methods to construct empirical models relating the measured spectrum to the target's attribute, e.g., carbon.

NIR measurements do not require any sample preparation and can be made under field moist conditions. Two systems for in-situ assessments are being developed, both measuring diffuse reflectance through a sapphire window in direct contact with the soil. One system, mounted on a tillage tool, measures carbon variation across a field at a fixed depth (typically $5-$ to $15-\mathrm{cm}$ subsurface). The second uses a probe to measure vertical profiles from the surface down to 30 $\mathrm{cm}$ or more. 
Mid Infra-Red Spectroscopy (MIDR)

James B. Reeves III

USDA, Environmental Management and By-Products Lab

Beltsville MD

For intensive environmental sampling, the present methods for analyzing soil $\mathrm{C}$, such as combustion, are too time consuming and expensive even for determining total-C, much less for the added determination of forms of $\mathrm{C}$ (inorganic, organic, labile, charcoal, etc.). New, rapid, and accurate methods are needed. One such method is diffuse reflectance infrared Fourier transform spectroscopy (DRIFTS) that entails irradiating a ground sample with mid-infrared radiation and collecting the resulting diffusely reflected radiation. Sample preparation is the same as for conventional methods, such as combustion, but since only one spectrum per sample is needed, multiple analyte determinations can be obtained simultaneously. Further, using an auto-sampler allows at least 360 samples per day to be analyzed compared to perhaps 100 or so by combustion for a single analyte. Research demonstrated that DRIFTS can determine within two minutes a wide variety of soil $\mathrm{C}$ fractions (organic-, inorganic- and total-C; various measures of biomass or bioactive $\mathrm{C}$ and $\mathrm{N}$; organic-N, free amino acids and sugars, among others) in a sample, and with greater accuracy than near-infrared reflectance spectroscopy (NIRS). Thus, DRIFTS can rapidly and simultaneously provide information on a wide range of C-based soil components, thereby maximizing the ability to quickly and inexpensively evaluate both soil $\mathrm{C}$ content and composition. However, several aspects of the spectral analysis of soils need further research: 1. Can one calibration be used to analyze all soil samples for any given analyte, and, if not, how must they be divided? 2. Which method, NIRS or DRIFTS, better allows the transfer of calibrations between the same and dissimilar instruments? 3. Can DRIFTS be used for in situ field measurements? 4. Can filter-based instrumentation be developed to facilitate the rapid, inexpensive, and on-site determination of soil $\mathrm{C}$ content and composition? 


\title{
Rapid Identification and Quantification of Soil Organic Carbon Forms Using Pyrolysis Molecular Beam Mass Spectrometry
}

\author{
K. A. Magrini ${ }^{1}$, M. F. Davis ${ }^{1}$, R. Follett ${ }^{2}$, E. Preussner ${ }^{2}$, J. Kimble ${ }^{3}$, C. M. Hoover ${ }^{4}$ \\ ${ }^{1}$ National Renewable Energy Laboratory; 1617 Cole Blvd., Golden, CO 80401, \\ ${ }^{2}$ USDA-ARS, 2150 Centre Avenue, Building D, Suite 100, Fort Collins, CO 80526- 8119 \\ ${ }^{3}$ USDA-NRCS (Retired), 7220 S. Hampton Rd., Lincoln, NE 68506 \\ ${ }^{4}$ USDA Forest Service, Northeastern Research Station, 271 Mast Road, PO Box 640, \\ Durham, NH 03824
}

Rapid quantitative analysis of the soil's carbon forms is required for assessing and monitoring managed agricultural and forest soils to establish carbon sequestration baselines, uptake, and retention. Present studies do not meet this need; instrumentation and methods must be developed to measure SOC inventories. We use analytical pyrolysis coupled with molecular beam mass spectrometry (py-MBMS) and multivariate statistical analysis to quantify SOC in wellcharacterized agricultural and forest soils. This rapidly pyrolyzes volatile components that are quenched in a molecular beam introduced into a triple quadrupole mass spectrometer for analysis. Samples are 100-500 mg, and typical analysis time from 3-5 minutes. Py-MBMS analysis of periodically disturbed forest soils easily distinguishes their chemistry, depths, and location from mass spectral signatures. Multivariate statistical analysis of mass spectral and independent characterization data of Midwestern agricultural soils demonstrate that carbon in the particulate organic matter-, mineral-, and microbial biomass- fractions can be quantitated. We used this technique to show that eighteen-year old agricultural soils in the Conservation Reserve Program agricultural soils have not reached native SOC or total carbon content. We are developing data based multivariate models to predict carbon content in soil fractions thereby to assess carbon sequestration pathways and progress. 


\title{
Soil Carbon Analysis by Inelastic Neutron Scattering
}

\section{Lucian Wielopolski, Sudeep Mitra, Oded Doron, and Yue Zhang.}

\author{
Brookhaven National Laboratory \\ Environmental Sciences Department \\ Upton, NY 11973
}

The unabated rise in atmospheric $\mathrm{CO}_{2}$ and the consequential changes in climate have strengthened the resolve of the Office of Science (SC), U.S. Department of Energy (DOE), to improve understanding of terrestrial carbon processes (TCP) that would assist in carbon-cycle predictions related to climate change. Improved quantification of soil carbon content and its components are essential for 1) better understanding of the belowground carbon processes, 2) resolving the missing carbon sink in the ecosystem, 3) improving carbon measurement with reduced error for large fields that are better suited for up-scaling, and, 4) obtaining data on temporal changes in soil carbon stocks for trading carbon credits through the Chicago Climate Exchange or the industry. Soil is a complex three-phase matrix convoluted with organic and inorganic matter containing living and dead organisms, aggregated into a system whose belowground activities are invisible to human eye. When the "black box" of this soil mélange is dissected for study, its function is altered [Johnston et al., 2004]. This, compounded by the physical variability in the soil's composition, presence of stones, debris, and litter are only part of the complexities inherent in soil measurements. Belowground processes presently are assessed both directly, by taking samples from the field into a laboratory for chemical analysis, and, indirectly, by eddy covariance towers measuring differences in $\mathrm{CO}_{2}$ fluxes to estimate net terrestrial gains or loses in carbon. Lal et al.'s book [2001] discusses the various considerations, issues, and errors associated with gathering soil samples and preparing them for CarbonHydrogen-Nitrogen (CHN) dry combustion chemical analysis. Eddy covariance methodology, its characteristics and limitations, are well described on the AmeriFlux web site. These two basic current state-of-the-art approaches established a few decades ago represent time- and spaceaveraged point measurements that are being extrapolated to regional-, national-, and globalscales. It is hard to envision how current sampling practices for the CHN analysis of soil will suddenly expand significantly to cover the new large areas required for strengthening our databases, or, for that matter, by increasing adequately the number of AmeriFlux towers. With marginal improvements over time, the scope of these methods clearly is limited, and they are becoming very inadequate for present-day and future needs to quantitatively determine transient and steady-state $\mathrm{C}$ levels in soil.

INS, one of the newly promoted analytical tools for measuring carbon in soil, is a nuclear method based on fast $14 \mathrm{MeV}$ neutrons interacting with nuclei of the soil elements via inelastic-, elastic-, and capture- reactions, inducing the emission of elemental gamma rays. The capture reactions take place after multiple scatterings of the fast neutrons that slow them down to thermal energies where they are captured and stimulate the emission of prompt gamma rays. These processes occur very fast, in microseconds, thus facilitating stationary and scanning measurements. An array of NaI detectors, mounted beside a neutron generator, provides for 
multi-elemental quantitative gamma ray spectroscopy [Wielopolski et al., 2003, Wielopolski et al., 2005]. Fig.1 shows two typical gamma ray spectra; one acquired during the neutron pulse representing gamma rays induced by inelastic neutron scattering events, INS spectrum, and two, a gamma ray spectrum acquired in between the neutron pulses representing thermal neutron capture, TNC events. The linearity of the system's response was tested against carbon concentrations in synthetic soils of sand mixed homogeneously with various amounts of carbon. Two calibration lines were derived (Fig.2); one representing the total net carbon counts in the carbon peak area, resulting in a very high intercept, and the second calibration line representing
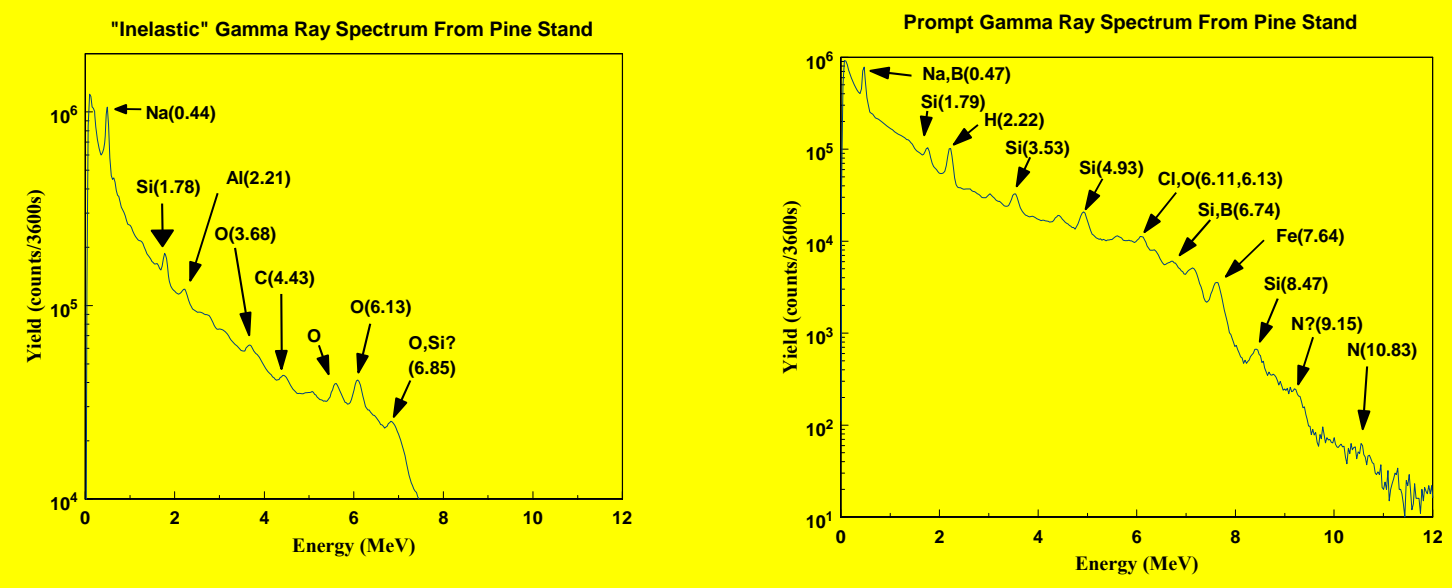

Figure 1. Two gamma ray spectra measured in soil due to inelastic events, on the left, and due to thermal neutron capture events, to the right,

the same counts as the first one but corrected for $\mathrm{Si}$ interferences due to escape peak in the $\mathrm{Si}$ thermal capture $4.93 \mathrm{MeV}$ peak and due to cascade of the 1.78 gamma ray in the inelastic spectrum. The corrected calibration line intercepts the $\mathrm{Y}$ axis much closer to zero and it parallels the uncorrected line.

There are few unique features in the INS system that sets it apart from the other emerging modalities. First, it uses a highly penetrating incident radiation of $14 \mathrm{MeV}$ neutrons and, similarly, detects high energy $4.4 \mathrm{MeV}$ gamma rays emanating from carbon nuclei, thus enabling sampling large soil volumes of about $0.3 \mathrm{~m}^{3}$ to an approximate depth of $25 \mathrm{~cm}$. The sampled volumes are resolved using Monte Carlo calculation simulating the INS system in its basic configuration. Boundaries of the volumes containing 90-, 95-, and 99-\% of the carbon signal reaching the detector are plotted in Fig. 3. The basic parameters of these volumes are summarized in Table 1 suggesting that the practical sampling depth is about $25 \mathrm{~cm}$.

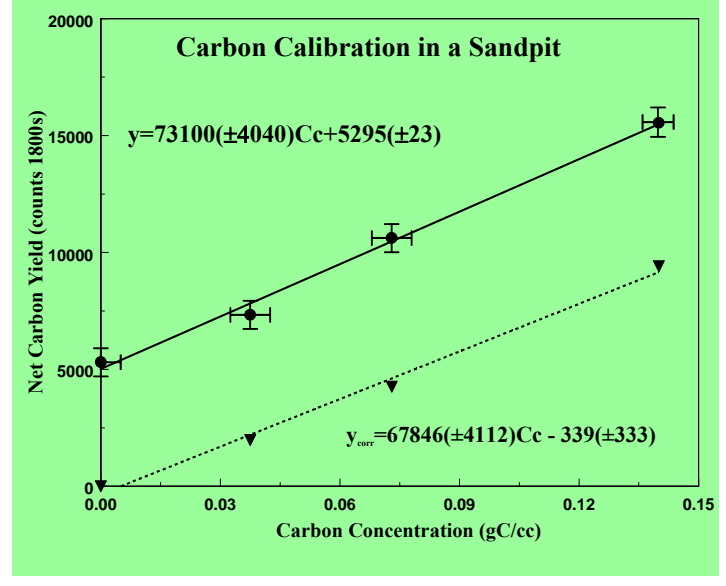

Figure 2. Calibration lines for the INS system using gross net counts in the carbon peak, upper line, and for the carbon peak counts, lower line, corrected for interferences. 


\begin{tabular}{|c|c|c|c|c|}
\hline \multicolumn{5}{|c|}{ Table 1 Parameters resulting from the } \\
calculated volumes for a homogeneous carbon \\
distribution in soil \\
\hline $\begin{array}{c}\text { \% } \\
\text { Total } \\
\text { Response }\end{array}$ & $\begin{array}{c}\text { Depth } \\
(\mathrm{cm})\end{array}$ & $\begin{array}{c}\text { Footprint } \\
(\mathrm{m} 2)\end{array}$ & $\begin{array}{c}\text { Volume } \\
(\mathrm{m} 3)\end{array}$ & $\begin{array}{c}\text { Mass } \\
(\mathrm{kg})\end{array}$ \\
\hline 90 & 25 & 2.400 & $\mathbf{0 . 2 3 3}$ & 325.66 \\
\hline 95 & 31 & 3.345 & $\mathbf{0 . 3 7 3}$ & 522.18 \\
\hline 99 & 44 & 7.069 & $\mathbf{0 . 7 8 9}$ & 1105.29 \\
\hline
\end{tabular}

The second unique feature is that it hovers about $30 \mathrm{~cm}$ above the ground and can be used in a stationary manner or be towed over large areas non-destructively. The third feature is that the error of the measurement can be reduced arbitrarily small and is proportional to the square root of the counting time. Finally, a complete response function of the INS system can be derived from basic principles using fundamental parameters and use for various simulations and system calibration. Fig. 4 shows an alpha prototype of the INS system placed over a pit of synthetic soils.

AmeriFlux, http://public.ornl.gov/ameriflux /

Johnston CA., P. Groffman, DD. Breshears, ZG. Cardon, W.

Currie, W. Emanuel, J. Gaudinski, RB. Jackson, K. Lajtha, K. Nadelhoffer, D. Nelson Jr., W Mac Post, G. Retallack, and L. Wielopolski, 2004. Carbon cycling in soil. Front. Ecol. Environ. 2(10); 522-528.

Lal R., JM. Kimble, RF. Follett, and BA. Stewart, 2001. Assessment Methods for Soil Carbon, Lewis Publisher.

Wielopolski L., Mitra S., Hendrey G., Rogers H., Torbert A., and Prior S., 2003. Non-Destructive In situ Soil Carbon Analysis: Principle and Results. Proceedings of the Second Annual Conference on Carbon Sequestration, May 3-5, Article No. 225.

Wielopolski L., S. Mitra, K. Johnsen, F. Sanchez, A. Torbert, S. Prior, H. Rogers, J. Ma, G. Hendrey, and R. Oren, 2005. A non-invasive method of detecting carbon in soil: comparison with soil cores and excavations. Proc. of the Fourth Annual Conference on Carbon Sequestration, May 2-5, Article \#56, (http://www.carbonsq.com/proc-search2004.cfm).

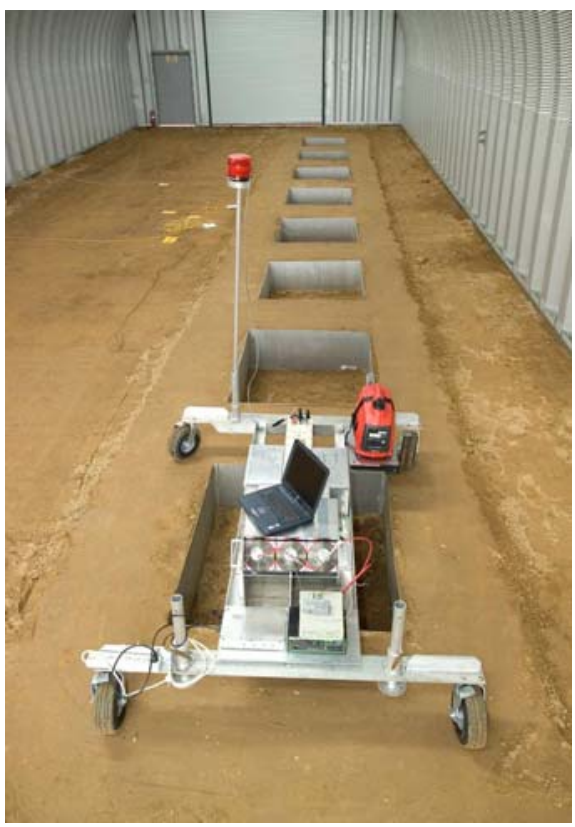

Figure 4 A mobile INS system over a pit for synthetic soils. 


\section{The Common Approach for Measuring Soil Carbon Amounts}

\section{Achim Dobermann}

Dept. of Agronomy and Horticulture, University of Nebraska P.O. Box 830915, Lincoln, NE 68583-0915

The classical approach for quantifying soil $\mathrm{C}$ amounts is a combination of destructive soil sampling and laboratory analysis for determining soil bulk density and the mass fraction of soil $\mathrm{C}$ in the sample. Due to the numerous steps involved, large variations in the accuracy and precision of estimated soil $\mathrm{C}$ amounts may occur. Key steps and issues to consider include:

1. Decide on a sampling strategy. A sample being analyzed for bulk density and soil C concentration may represent a widely varying area or amount of soil:

a. Time of sampling.

b. No. of samples collected (no. of locations per area).

c. Core diameter used.

d. Sampling depths: most common in soil C studies are depths of 0-5, 5-15, and 15-30 $\mathrm{cm}$, but deeper sampling may be required in systems with deep tillage.

e. Bulking strategies: how many cores are combined into a composite sample?

2. Measure soil bulk density in undisturbed soil samples:

a. Core method (most common) or other methods (clod method, excavation method). How many cores per area?

b. Weigh before and after drying at $105{ }^{\circ} \mathrm{C}-$ several potential weighing errors.

c. For accurate calculation, measure the exact actual diameter of the cutting tip of the push probe used (may vary by $\pm 10 \%$ among probes of the same type) and account for gravel, stones, and fragments $>2 \mathrm{~mm}$.

3. Process and grind soil samples for $\mathrm{C}$ analysis:

a. Dry at about $40^{\circ} \mathrm{C}$. Avoid contamination.

b. Remove visible organic particles and rocks - removal of undecomposed organic matter may vary widely among laboratories and people doing such work.

c. Grinding for analysis: crush and grind the entire sample to $2 \mathrm{~mm}$ size followed by fine-milling (100-mesh) of a smaller sub-sample for soil $\mathrm{C}$ analysis.

4. Determine the concentration of soil C:

a. In non-calcareous soils, soil organic $\mathrm{C}(\mathrm{SOC})$ is assumed to be equal to the total soil $\mathrm{C}$ concentration. Common measurement methods include:

i. Dry combustion (CN analyzer): most accurate, requires fine-milled sample.

ii. Wet digestion (Walkley-Black): phased out due to numerous problems.

iii. Loss on ignition (LOI): Semi-quantitative method because it measures soil organic matter, not SOC. Conversion to SOC is problematic.

b. In calcareous soils, inorganic soil $\mathrm{C}$ must be determined and subtracted from total soil C to obtain SOC. Results may vary depending on sample treatment.

c. Using fine-milled sampled and dry combustion analysis of soil $\mathrm{C}$, coefficients of variation for repeated measurements of the same sample are typically 1 to $3 \%$. 
5. Calculate the total amount of soil $\mathrm{C}$ in the soil profile or depth of interest, expressed, for example, in $\mathrm{g} \mathrm{C} \mathrm{m}^{-2}$ of $\mathrm{Mg} \mathrm{C} \mathrm{ha}^{-1}$ for a certain soil depth or mass of soil:

a. Soil C amount expressed on a constant soil depth basis (= constant soil volume): this is only appropriate if the whole soil profile is sampled or no significant difference in bulk density exists between samples that are compared.

b. Soil $\mathrm{C}$ amount expressed on constant soil mass basis $(=\mathrm{C}$ amount in the same equivalent soil dry mass): this is required if only a relatively shallow part of the soil profile is sampled and when significant differences in bulk density occur between samples that are compared with each other. Two methods are most widely used for equivalent mass basis expression of soil C (Ellert and Bettany, 1995; Gifford and Roderick, 2003). In mineral soils, typical reference amounts of soil dry mass for expressing soil C amounts are $200 \mathrm{~kg}$ dry soil $\mathrm{m}^{-2}\left(\sim 0-15 \mathrm{~cm}\right.$ depth) or 400 dry soil $\mathrm{m}^{-}$ 2 ( 0-30 cm depth).

c. In many published studies, particularly older long-term experiments, lack of measurements does not allow accurate monitoring of soil $\mathrm{C}$ amounts over time. Missing data are often substituted by regression and soil $\mathrm{C}$ is often not reported on equivalent soil mass basis. This can lead to large errors in the conclusions drawn. In cropping systems that lead to an increase in bulk density (e.g., no-till relative to conventional tillage), failure to express soil $\mathrm{C}$ on equivalent soil dry mass basis always leads to a biased estimate, i.e., an apparently greater soil $\mathrm{C}$ accumulation in the treatment in which bulk density is highest.

6. Calculate the annual soil C sequestration rate (e.g., $\mathrm{g} \mathrm{C} \mathrm{m}^{-2} \mathrm{yr}^{-1}$ or $\mathrm{Mg} \mathrm{C} \mathrm{ha}^{-1} \mathrm{yr}^{-1}$ ):

a. As the absolute change in soil $\mathrm{C}$ over time. This should be the Gold Standard, but it requires accurate measurements of bulk density and soil $\mathrm{C}$ over time.

b. Relative change in soil $\mathrm{C}$ at a certain point in time, i.e., by comparing a treatment with a certain reference treatment or baseline. Common approaches are to calculate the relative change in soil $\mathrm{C}$ as (1) the difference between treatment $\mathrm{A}$ and treatment B measured after $n$ years or (2) the difference between treatments A and B measured after $n$ years divided by the difference between treatments A and B measured at the beginning of the study. This method has been most widely used in the literature, but it is also prone to errors. Ratio estimators depend on the values measured for both treatments, including all of the potential error sources. A calculation may yield a positive relative change in soil $\mathrm{C}$ even though both treatments may be loosing soil $\mathrm{C}$ on an absolute basis.

c. Rates of change in soil $\mathrm{C}$ differ for different time periods, making it difficult to derive a reliable value for an "average" rate of soil C sequestration or loss over time.

\section{References}

Ellert, B.H., and J.R. Bettany. 1995. Calculation of organic matter and nutrients stored in soils under contrasting management regimes. Can. J. Soil Sci. 75:529-538.

Gifford, R.M., and M.L. Roderick. 2003. Soil carbon stocks and bulk density: spatial or cumulative mass coordinates as a basis of expression? Global Change Biol. 9:1507-1514. 


\section{Measuring and Predicting Soil Carbon Changes at the Field Scale}

\section{R. César Izaurralde ${ }^{1}$, Allison M. Thomson ${ }^{1}$, and Aaron Rappaport ${ }^{2}$ \\ ${ }^{1}$ Joint Global Change Research Institute, PNNL and Univ. of Maryland ${ }^{2}$ Private Consultant}

To become an effective tool for climate change mitigation, soil carbon sequestration (SCS) will have to be deployed globally over the course of several decades in this century. The implementation of SCS practices is likely to occur under diverse landscapes, soils, climate, and land management. Thus, accurate but comparable methodologies will have to be developed to monitor soil carbon changes at different spatiotemporal scales. This presentation will examine different methodologies available for measuring and predicting soil carbon changes at the field scale. These methodologies are based primarily based on direct measurements and simulation modeling of soil carbon change. Several aspects of methodologies for detecting soil carbon changes under field conditions will be discussed such as spatial design, sampling size, sampling depth, ancillary measurements, laboratory procedures, and calculations. The discussion on simulation modeling will center on the accuracy and requirements of models for the prediction of soil carbon changes at field and regional levels. The integration of direct measurements with simulation modeling and other methodologies will also be discussed. 


\title{
Monitoring Spatial and Temporal Carbon Changes in the Field
}

\author{
Charles W. Rice \\ Department of Agronomy \\ Kansas State University \\ Manhattan, KS
}

Carbon storage in soil has significant implications on atmospheric $\mathrm{CO}_{2}$. On a global scale, soils are important both as a source and sink of $\mathrm{C}$. Enhanced sequestration and reduced emissions become important in soil $\mathrm{C}$ management. However detecting changes in soil $\mathrm{C}$ is often difficult because of soil characteristics and the inherent variability both in time and space. Measuring changes in soil $\mathrm{C}$ is further complicated by the large amount of $\mathrm{C}$ that is relatively inactive on annual time scales. Thus it is difficult to detect changes given the large amount of spatial variability in the total soil $\mathrm{C}$ introduced by different environmental conditions and histories.

Traditionally, changes in SOC induced by management have been measured in long-term experiments by soil sampling and laboratory determinations. These data have been instrumental in the development of models able to predict SOC changes that result from various climate-soilmanagement combinations. A cost effective protocol is needed to monitor compliance for the implementation of mitigation contracts.

One means to reduce variability is to establish spatially explicit sites for monitoring change in soil carbon. However, questions of proper sample size, sample number, and depth increments need to be evaluated. A standard protocol for field sampling and laboratory analyses is needed for a soil $\mathrm{C}$ inventory. There are several issues that need to be considered to develop an effective and cost efficient protocol. The number and locations of monitoring sites need to be considered. Proper selection of depth increments is needed. Many procedures recommend 0-5, 5-15, 15-30, and 30-45 cm depending upon the soil and management. The small depth increments near the surface often detect changes as a result of soil and plant management. However, deeper depths are required to insure carbon is not a result of redistribution. Measuring changes in mass or volume of the soil are required to determine mass of carbon and is a significant source of error.

The value of microsites for monitoring temporal change reduces the lateral spatial variability associated with soil measurements. Microsites also allow for new technologies to be employed as they become available whether it is models or new in situ instrumentation. Calibration, validation and error estimates are needed to implement any new protocol or instrumentation. With careful thought existing technologies will allow detection of changes in soil carbon that can be monitored and verified. New instrumentation will decrease costs and potentially reduce error. 


\title{
Strategies for Monitoring and Mapping of Soil $\mathrm{C}$ in Large Fields
}

\author{
Achim Dobermann \\ Dept. of Agronomy and Horticulture, University of Nebraska \\ P.O. Box 830915, Lincoln, NE 68583-0915
}

To obtain reliable estimates of soil $\mathrm{C}$ sequestration rates under different management, small changes in soil $\mathrm{C}$ must be quantified against a high background level of already existing $\mathrm{C}$ in the soil profile. Estimation of the mean soil $\mathrm{C}$ amount in an area is required in research on $\mathrm{C}$ budgets at landscape level as well as for field verification of soil $\mathrm{C}$ sequestration. Earlier studies have focused on monitoring in small plots and at grassland sites (Garten and Wullschleger, 1999; Conant and Paustian, 2002; Conen et al., 2003), where soil $\mathrm{C}$ variability tends to be less than in large crop production fields. We used data of detailed soil $\mathrm{C}$ surveys conducted at three sites in Nebraska (Verma et al., 2005; Simbahan et al., 2006) to quantify sampling requirements and the attainable detection limits for measuring a change in the mean soil $\mathrm{C}$ amount in 0 to $0.3 \mathrm{~m}$ depth over time. Using only measured soil $\mathrm{C}$ values, 150 to 200 samples per field were required to be able to detect a change in soil $\mathrm{C}$ of $5 \mathrm{Mg} \mathrm{C} \mathrm{ha}^{-1}$ (Fig. 1) by random re-sampling. Assuming average potential $\mathrm{C}$ sequestration rates of 25 to $50 \mathrm{~g} \mathrm{C} \mathrm{m}^{-1} \mathrm{yr}^{-1}$, it would take 10 to 20 years to be able to detect such a change. When available secondary information was used in a regression estimator (Brus, 2000) of mean soil C, only 70 to 90 samples were required for a minimum detectable difference (MDD) of $5 \mathrm{Mg} \mathrm{C} \mathrm{ha}^{-1}$ or, with higher sampling intensity, the detection limit could be decreased to $3 \mathrm{Mg} \mathrm{ha}^{-1}$ (Fig. 1). This is equivalent to about 6 to 12 years of soil $\mathrm{C}$ sequestration (Fig. 1).
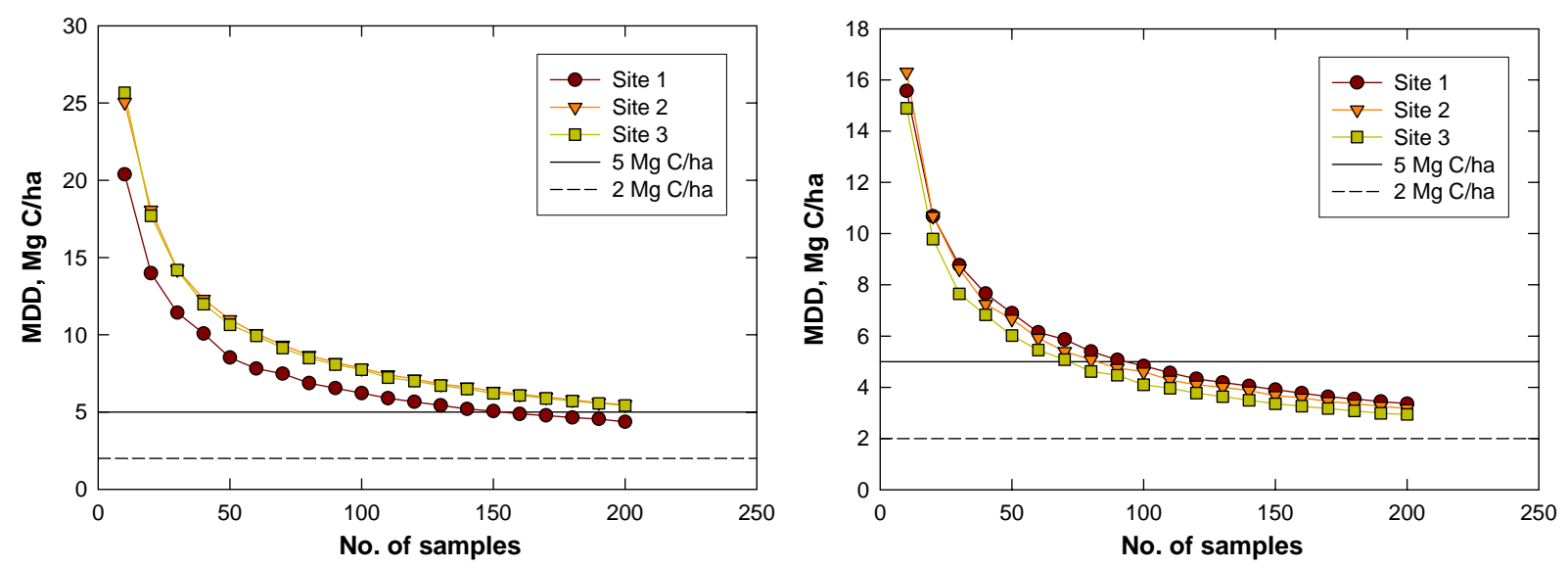

Figure 1. Influence of sample size and estimation method on the mean detectable difference in soil $\mathrm{C}(\mathrm{Mg} \mathrm{C} / \mathrm{ha}$ in 0-0.3 m depth). Left: classical statistical approach based on measured variance of soil $\mathrm{C}$; Right: modified approach using a regression estimator of mean soil $\mathrm{C}$, based on measured soil $\mathrm{C}$ and exhaustive secondary information (EC, remote sensing).

An alternative and cheaper strategy is to collect and analyze fewer samples per field, but each composed of a larger number of soil cores collected from multiple locations in the field. A 
sampling strategy using 20 composite samples per field and 20 sub-samples per composite sample in combination with a regression estimator reduced the MDD to $2.5 \mathrm{Mg} \mathrm{C} \mathrm{ha}^{-1}(3-4 \%$ relative change in soil $\mathrm{C}$, or roughly 5 to 10 years of potential $\mathrm{C}$ sequestration). The major implication from our analysis is that it is not possible to detect small changes in soil $\mathrm{C}$ occurring over time periods of less than five years through random re-sampling. Carbon credit and trading programs that only require short-term commitments to management changes are thus not verifiable with affordable means. A smaller MDD can only be achieved by geo-referenced paired sampling, i.e., repeated sampling of the same locations or monitoring plots within a field.

Detailed maps of soil C amounts are primarily of interest for research studies. Our work has demonstrated that the use of spatially-dense secondary information such as a digital elevation models (DEM), on-the-go sensed electrical conductivity (Veris sensor), bare soil imagery, and digital soil type maps in combination with destructive soil sampling greatly increases the accuracy of soil $\mathrm{C}$ maps. Multivariate regression kriging that utilized such secondary information increased the accuracy of soil $\mathrm{C}$ maps by 16 to $38 \%$ compared to ordinary kriging of measured soil $\mathrm{C}$ alone (Simbahan et al., 2006). We have also developed a new approach on dual use of secondary information in soil C mapping (Simbahan and Dobermann, 2006). Sampling optimization based on secondary information and re-use of the secondary information in regression kriging greatly increased the accuracy of soil $\mathrm{C}$ maps and allowed a significant reduction in sample size without loss of information.

\section{References}

Brus, D.J. 2000. Using regression models in design-based estimation of spatial means of soil properties. Eur. J. Soil Sci. 51:159-172.

Conant, R.T., and K. Paustian. 2002. Spatial variability of soil organic carbon in grasslands: implications for detecting change at different scales. Environ. Pollut. 116:127-135.

Conen, F., M.V. Yakutin, and A.D. Sambuu. 2003. Potential for detecting changes in soil organic carbon concentrations resulting from climate change. Global Change Biol. 9:1515-1520.

Garten, C.T., and S.D. Wullschleger. 1999. Soil carbon inventories under a bioenergy crop (Switchgrass): measurement limitations. J. Environ. Qual. 28:1359-1365.

Simbahan, G.C., and A. Dobermann. 2006. Sampling optimization based on secondary information and its utilization in soil carbon mapping. Geoderma doi:10.1016/j.geoderma.2005.07.020

Simbahan, G.C., A. Dobermann, P. Goovaerts, J.L. Ping, and M.L. Haddix. 2006. Fineresolution mapping of soil organic carbon based on multivariate secondary data. Geoderma doi:10.1016/j.geoderma.2005.07.001

Verma, S.B., A. Dobermann, K.G. Cassman, D.T. Walters, J.M.H. Knops, T.J. Arkebauer, A.E. Suyker, G.G. Burba, B. Amos, H.S. Yang, D. Ginting, K.G. Hubbard, A.A. Gitelson, and E.A. Walter-Shea. 2005. Annual carbon dioxide exchange in irrigated and rainfed maizebased agroecosystems. Agric. Forest Meteorol. 131:77-96. 


\title{
Unique Aspects of Sampling Soil C in Forest Systems
}

\author{
Kurt H. Johnsen \\ USDA Forest Service Southern Research Station \\ PO Box 12254 \\ Research Triangle Park, NC 27709
}

\begin{abstract}
Forest systems offer extreme challenges for estimating soil C. Even "homogeneous" pine plantations near harvest age display high spatial variation, vertically and horizontally, requiring large sample sizes to detect statistically significant differences among treatments or sites. Unlike agriculture sites, many forest soils have large volumes of coarse fragments; at the Duke FACE site, approximately $10 \%$ of the soil volume is composed of stones and values ranged from 0 to 48 percent. Course fragments often make random sampling impossible and course fragment volume needs to be estimated to scale $\mathrm{C}$ concentration values to the stand level. Following a disturbance, root systems decay and can cause a large pulse in soil $\mathrm{C}$, thus temporal variation needs to be considered when estimating $\mathrm{C}$ pools over time. Due to transpiration, forests greatly impact water table depth. For example, clear-cutting a coastal plain pine stand raised the water table from approximately -2 meters to frequent periods of standing water. In stands such as these, site preparation usually includes displacing soil mechanically, which creates three distinct microsites including the raised bed, a trough where the soil was removed and the relatively undisturbed inner beds. The beds have a partly inverted soil $\mathrm{C}$ profile and are highly porous with heterogeneous coarse woody debris incorporated belowground. The troughs typically have the lowest $\mathrm{C}$ concentrations, have higher bulk density, and are often inundated with water in the early stages of stand development. The impacts of bedding are evident even at rotation, which increases the spatial variation in soil $\mathrm{C}$ and further complicates sampling.
\end{abstract}




\section{Effects of Sampling Volume on Error}

\section{Harold van Es \\ Dept. of Crop and Soil Sciences, Cornell University.}

Each measurement procedure has associated problems with analyses and reliability of result, and appropriate methodologies will, in part, result from the researcher's choices. The dependence of the measured response on the scale of observation (also called sample support) seriously challenges all soils research. With increasing size of the soil volume or area that is measured, variance generally decreases. Sometimes, the mean response may also be affected. These relationships can be explained through the concept of the representative elementary volume (REV). Microscopically, soil properties and mass flow processes are highly variable. For example, at this small scale, a sample may have a $\mathrm{C}$ content ranging (perhaps) from 0 to 0.5 $\mathrm{g} / \mathrm{g}$, depending on whether a mineral, a piece of organic matter, or fractions of both are sampled. With increasingly larger sample volumes, the $\mathrm{C}$ content becomes equivalently less variable as observations become macroscopic and spatially integrated. The definition of an REV, therefore, is the smallest volume of soil that can represent the range of microscopic variations. Although the REV is an elusive concept, in practice it offers guidance on identifying an acceptable measurement scale.

With increasingly larger sample volumes, the frequency distribution logically transforms with changes in the variance and, in some cases, the mean. In addition, the shape of this frequency distribution may alter towards normality, as defined by the Central Limit Theorem. Often, non-Gaussian behavior may indicate an inadequate scale of observation. Besides measuring at an adequately representative scale, researchers must balance the costs of measurements at different scales. Increasing the measurement domain generally yields more precise results, but also may be more costly and time-consuming, or prevent a good distribution of samples in the domain. Measurements on smaller volumes may be more variable, but many more and better distributed, observations can be made. Generally, a scale can be defined that reasonably balances the needs for representation and precision e with logistical ease. 


\title{
Soil Carbon Analysis in Large Fields Using a Scanning System
}

\section{Lucian Wielopolski, Sudeep Mitra, Yue Zhang, A. Torbert ${ }^{*}$ and S. Prior*}

\author{
Brookhaven National Laboratory \\ Environmental Sciences Department \\ Upton, NY 11973 \\ *USDA-ARS National Soil Dynamic Lab
}

Auburn AL

One of the issues hindering the implementation of carbon-credits trading protocols and lack of comprehensive databases required for the models that determine the fate of carbon across North America is the need for frequent and repetitive soil analysis over large areas. However, this is not possible at present due to lack of instrumentation for carbon analysis in soil that is inexpensive, fast, and can scan large areas in a non-destructive manner. A limiting factor in comprehensively evaluating large fields for carbon with the current state-of-the-art equipment is the need for analyzing soil core samples in a laboratory or, alternatively, using AmeriFlux towers. These procedures are labor-intensive, involving many steps, time consuming and prohibitively expensive when there are large numbers of samples. Therefore, there is an emerging need for novel types of instrumentation for in situ soil carbon analysis that, preferably, are nondestructive, yet able to contiguously scan large areas. The INS system, introduced ibid, p 39, satisfies most of the requirements. The instrument is based on gamma- ray spectroscopy induced by Inelastic Neutron Scattering. This nuclear method directs fast $14 \mathrm{MeV}$ neutrons at the soil where they undergo inelastic neutron scatterings stimulating characteristic gamma ray emission from the soil's elements including carbon. The time intervals from the time of neutron emission to detection and recording of carbon gamma rays are very short, in the range of microseconds. In comparison with typical tractor speeds in the field of about five $\mathrm{km} / \mathrm{hr}$, or twice that, the system is virtually stationary relative to the nuclear events taking place. Fig.1 depicts the gamma rays' temporal characteristics induced by a neutron generator operating at $10 \mathrm{kHz}$ and $25 \%$ neutron duty cycle. It shows that a neutron cycle will be completed before the INS system moves, thus enabling using INS in a scanning mode and, since the system hovers over the soil, the scans are non-destructive.

The number of counts, $\mathrm{R}_{\mathrm{s}}$, recorded by a stationary INS system (the response function) can be expressed as a multiple integral given in Eq. 1 in which $\varphi_{\mathrm{n}}$ is the neutron flux at an

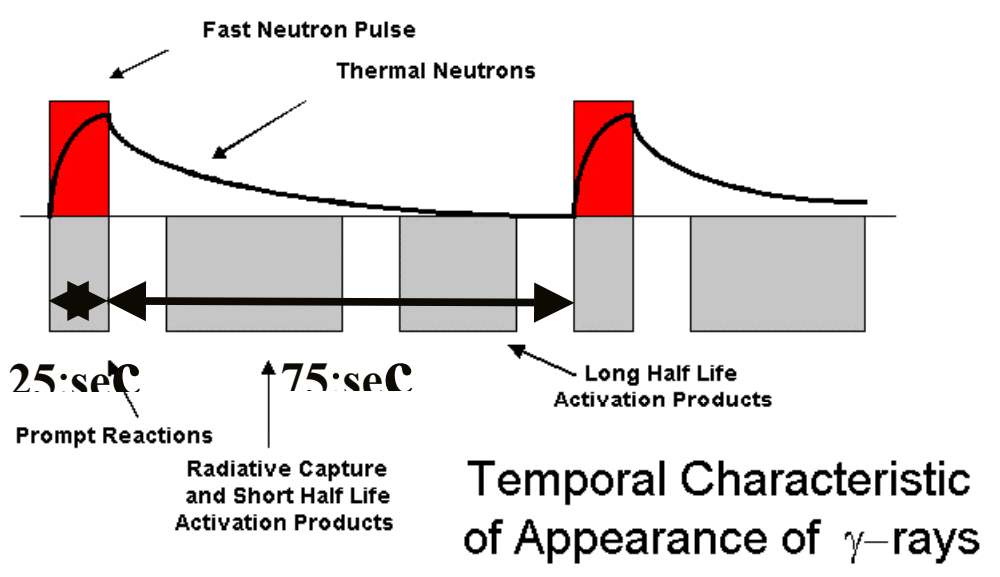

Figure 1. Temporal characteristics of gammarays induced by a neutron generator operating at $10 \mathrm{kHz}$ and $25 \%$ duty cycle. 


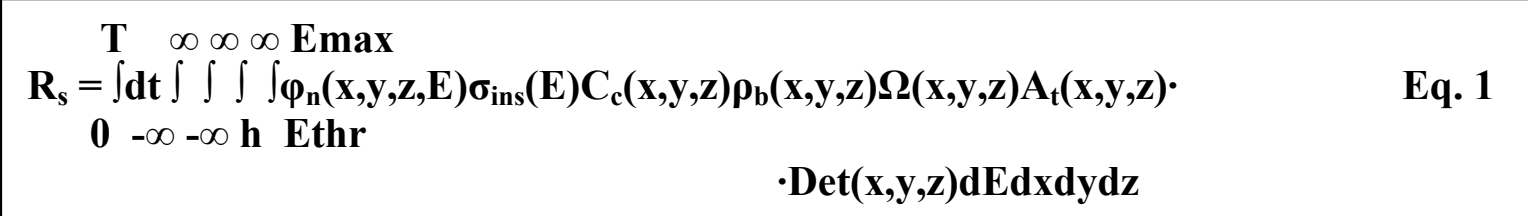

arbitrary point in soil, $\sigma_{\text {ins }}$ is the inelastic neutron scattering cross-section for carbon, $\mathrm{C}_{\mathrm{c}}$ is carbon concentration in the soil, $\rho_{\mathrm{b}}$ is the bulk density, $\Omega$ is the solid angle subtended by the detection system, $A_{t}$ is gamma rays attenuation in the direction of the detectors, and Det is the detection system response function. The integration is carried over the counting time $\mathrm{T}$ and the volume $\mathrm{V} \rightarrow \infty$. Thus we can simplify Eq. 1 and state that the INS signal $\mathrm{R}$ is proportional to counting time and sampled volume and for non-changing condition it remains constant, Eq. 2. Thus counting for shorter times but larger volumes the response should be the same. In a scanning

$$
\mathbf{R} \boldsymbol{\alpha} \mathbf{T} \cdot \mathbf{V}=\mathbf{c o n s t}
$$

Eq. 2

system some of the parameters in Eq. 1 are field dependent therefore scanning in $\mathrm{x}$ direction at speed $\mathrm{v}$ we can expressed them as:

$$
\varphi_{n}\left(x+x_{0}+v t, y, z, E\right) ; \quad C_{f}\left(x+x_{0}+v t, y, z\right) ; \quad \rho_{b}\left(x+x_{0}+v t, y, z\right) ; \text { and } A_{t}\left(x+x_{0}+v t, y, z\right)
$$

Thus, a response of a moving system is an integral of the stationary response (Eq. 1) over the entire field and we can write the mean field response per unit area is:

$$
\text { Mean Field Response }=\int F(X, Y, Z) R_{s}(X, Y, Z) d V / A \quad[g C / c m 2] \quad \text { Eq. } 3
$$

Where F represents field dependable parameters.

To demonstrate the scanning capability the INS system was mounted on a trolley moving on rails spanning a soil bin filled with homogenous soils, Fig. 2. Three bins with different soil compositions were measured statically at four spots and then scanned at various speeds; bin 1 at speed $v$, bin 2 at speed $2 \mathrm{v}$, and bin 3 at random speeds all of them for the same counting times as the static measurements. Table 1 summarizes the results. The mean static counts are the means of the four spots used in each bin.

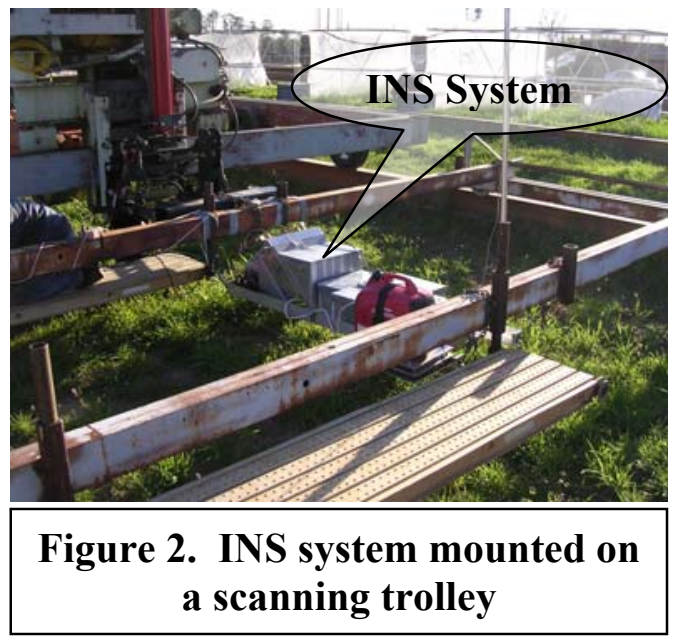




\begin{tabular}{|c|c|c|c|}
\hline \multicolumn{4}{c}{ Table 1 } \\
& $\begin{array}{c}\text { Mean Static } \\
\text { Counts }\end{array}$ & $\begin{array}{c}\text { Dynamic } \\
\text { Counts }\end{array}$ & $\begin{array}{c}\text { Difference } \\
\text { In SD }\end{array}$ \\
\hline $\begin{array}{c}\text { Hiwassee } \\
\text { Clay (V1) }\end{array}$ & 19,229 & 19,091 & $0.1 \sigma_{\mathrm{n}-1}$ \\
\hline $\begin{array}{c}\text { Vaiden Silty } \\
\text { Clay (2V1) }\end{array}$ & 26,783 & 25,564 & $0.8 \sigma_{\mathrm{n}-1}$ \\
\hline \hline $\begin{array}{c}\text { Hiwassee Sandy } \\
\text { Loam (Random V) }\end{array}$ & 30,449 & 31,117 & $0.7 \sigma_{\mathrm{n}-1}$ \\
\hline
\end{tabular}




\section{Calibration, Validation, and the Question of Outliers}

\section{David J. Brown \\ Montana State University}

The determination of soil organic C using Visible and Near-Infrared (VNIR) and Mid-Infrared (MIR) diffuse reflectance spectroscopy was powerfully and significantly advanced by using high-dimensional chemometric and data-mining techniques. Nevertheless, they must be used extremely carefully to reliably estimate prediction accuracy. This presentation, largely on reflection spectroscopy, will address three key issues: (i) Selecting suitable calibration and validation datasets for constructing models and predicting error estimation; (ii) identifying spectral outliers; and, (iii) identifying validation outliers.

Randomly selecting a holdout validation dataset is a common practice for soil analysis. However, samples generally are not randomly selected from the population, but are clustered by site and profile. In these cases, such random selection can significantly underestimate prediction error. I propose imposing a stricter requirement for geographic independence on the choice of validation samples.

Empirical calibrations can only be applied to "similar" samples, so the construction of soilspectral libraries requires that spectrally "different" samples are screened before laboratory analysis. Mapping in the spectral space of principal components usually identifies similar and different samples. However, this approach assumes that (i) all scaled spectral differences are equally important; and, (ii) soil reflectance spectra map to unique soil compositions.

Commercial chemometric software packages automatically remove poorly fit "outliers" from calibration and validation results. Consequently, many researchers excise poorly fit observations from their predictions. However, without independently confirming an error in coding or measurement, observations should never be removed from validation results, and only with great care from model calibrations. 


\section{Reference materials and synthetic soils}

\section{B.H. Ellert and H.H. Janzen}

Agriculture \& Agri-Food Canada Research Centre, $54031^{\text {st }}$ Avenue South, Lethbridge, Alberta T1J 4B1 CANADA

Chemical determination of the carbon content of soil must be among the earliest and commonest analysis used by investigators. Perhaps because soil $\mathrm{C}$ exists as a heterogeneous mixture of organic constituents, as well as charcoal-like materials and inorganic carbonates, a range of analytical techniques has been applied, but still uncertainty remains in the measurement scales. In the first part of our presentation, we review the availability of standard reference materials, or geostandards, with values for $\mathrm{C}$ content that may be relevant to harmonizing these scales. We emphasize powdered materials with a soil-like matrix, including soils, freshwaterand marine-sediments, rocks, and minerals. We also discuss the use of geostandards to verify the accuracy of conventional analyses of soil by automated combustion techniques, paying particular attention to the partitioning of total $\mathrm{C}$ between organic and inorganic forms. In the second part of the presentation, we share some of our experiences with 'synthetic' or reconstructed soils, prepared in small field plots. Originally, such experiments were devised to address questions about a soil's productive capacity, and for detecting temporal changes in soil C. Some of these approaches may be useful to evaluate novel ways to assess $\mathrm{C}$ concentrations in soil, and to estimate stocks. 


\section{Modeling Soil Carbon Sequestration over Large Areas}

\section{P.C. Doraiswamy ${ }^{1}$, E.R. Hunt ${ }^{1}$, R. C. Izaurralde $^{2}$, G. McCarty ${ }^{1}$ \\ ${ }^{1}$ U.S. Department of Agriculture, ARS, Hydrology and Remote Sensing Laboratory ${ }^{2}$ Pacific Northwest National Laboratory}

Evidence for global climate change intensified international efforts toward reducing anthropogenic $\mathrm{CO}_{2}$ sources and increasing the sink capacities of the terrestrial biosphere, particularly for agricultural ecosystems that may become substantial $\mathrm{CO}_{2}$ sinks under optimum management. Directly measuring changes in soil organic matter on regional scales, such as the U.S. Corn Belt, is complex because of spatial variability in climate, soil properties, and crop management practices, and requires enormous resources. Biogeochemical models offer valid alternatives for estimating long-term changes in soil carbon

We examined the impact of management on soil carbon sequestration in Central Iowa using the EPIC-CENTURY biogeochemical model to simulate the baseline level of soil carbon from soil survey data, and project changes in soil organic carbon (SOC) under different tillage practices for corn and soybean. First, we modeled a 25-year period (1970-1994) using conventional tillage practices, with state-wide average fertilizer inputs. NOAA NWS weather data drove the models. Initial SOCs reflected the soil's average organic-matter concentration and average bulk density from the STATSGO databases. Then different modeling scenarios were applied for 25 years (1996-2020): A, conventional tillage; B, mulch tillage; C, no-till method; and D, no-till with a rye crop. After 25 years of conventional tillage in a two-year maize-soybean rotation, the average carbon content was $60 \mathrm{Mg} \mathrm{C} / \mathrm{ha}, 2 \mathrm{Mg} \mathrm{C} / \mathrm{ha}$ less than initial conditions. In 2020, conventional tillage is predicted to reduce the average SOC another $10 \mathrm{Mg} \mathrm{C} /$ ha to establish an expected baseline.

With erosion turned off, simulated SOC in 2020 under continuous conventional tillage was $27 \%$ higher than the baseline. With mulch till, the SOC is $59 \mathrm{Mg} \mathrm{C} / \mathrm{ha}$, similar to starting conditions. However, compared to the expected baseline loss of carbon, mulch till sequestered $9 \mathrm{Mg} / \mathrm{ha}$, i.e., $300 \mathrm{~kg} \mathrm{C} \mathrm{ha}{ }^{-1}$ year $^{-1}$. No-tillage practices averaged $76 \mathrm{Mg} \mathrm{C} / \mathrm{ha}$, an absolute gain from 19962020 .

The amount of soil carbon sequestered represents increased inputs of residue and reduced loses from erosion. The former can be determined by remote sensing that will show the overall change and type of conservation tillage practices adopted by farmers. Importantly, however, the location of these practices strongly interacts with other variables such as erosion, so area-wide averages will not reliably indicate the yearly rate of carbon sequestration. 


\section{Cost Assessment of Standard Soil Sampling}

\section{H. Allen Torbert}

Recently, due to concerns regarding the potential for global change due to increased atmospheric $\mathrm{CO}_{2}$ levels there has been increased interest in evaluating the potential for $\mathrm{C}$ sequestration in soil. This has lead to interest in measuring changes in soil $\mathrm{C}$ due to changes in land management practices. In this paper, I discuss the potential costs of standard soil sampling and analysis for this purpose. To determine costs of sampling on a field scale, many methods used traditionally for establishing the soil's fertility can be employed, including methods to determine the places to sample and the number of sub-samples needed. However, additional considerations are required to fully assess soil $\mathrm{C}$ levels for addressing changes in $\mathrm{C}$ sequestration. The primarily concern is that soils are inherently variable and that with $\mathrm{C}$ sequestration, small differences are very important. Small changes can be very important because of the massive weight and volume involved in soil, with one ha of land at $7.5 \mathrm{~cm}$ soil depth containing approximately $1,120,000 \mathrm{~kg}$ of soil. Sample collection methods that can detect small changes in $\mathrm{C}$ concentration will be critical. To adequately measure soil $\mathrm{C}$ sequestration, sampling schemes and laboratory methods must be used that will enhance the probability of detecting potential changes in soil $\mathrm{C}$ status.

When looking at the typical soil profile, most of the $\mathrm{C}$ is found near the soil surface. Changes in the top 5 to $10 \mathrm{~cm}$ in soil $\mathrm{C}$ may be critical for accounting for $\mathrm{C}$ sequestration due to changes in land management. However, changes in land management will also change the distribution of $\mathrm{C}$ in the soil profile, and in the long run, the changes can be expected over time at increased soil depths. It will be important to not only account for the small changes in the soil surface, but also to examine the whole soil profile for small changes.

Traditional soil testing includes only one soil increment, generally $0-15 \mathrm{~cm}$. This depth is utilized because it is the plow layer for conventional production agriculture and the soil would general be physically mixed within this layer under traditional tillage conditions. Also, this is the layer were the most intense root activity occurs in most row crops. For traditional soil sampling for soil fertility recommendations, sampling in this zone gives the best results. However, when land management practices change so that the surface is not mechanically mixed as with conservation tillage or pasture, changes in soil $\mathrm{C}$ can occur at various depths within this 0-15 cm soil layer. Also, since even small changes can be important, it is not likely that soil sampled can be mixed adequately after sampling to detect these small changes that can be important for $\mathrm{C}$ sequestration calculations. While soil fertility measures can be best measured by looking where the greatest root activity occurs, with $\mathrm{C}$ sequestration it is important to also account for changes that may occur through the whole soil profile. Since the distribution of soil $\mathrm{C}$ is likely as land management practices change, it is important that increased soil depths be considered. This will entail additional cost for equipment that can sample deeper, and additional processing modalities to cut and maintain separate cores by depth.

Another problem inherent with soil sampling is spatial variability. To address this variability, traditional soil sampling utilizes composite sampling where multiple soil cores are collected and combined to provide one soil sample per area for analysis. Areas of the landscape with similar physical conditions are grouped and soil sampling is performed within these areas. 
These areas are recommended to be $<8$ to16 ha and 10 to 35 sub-samples are combined to provide one composite sample within this area. A much greater frequency across the landscape is recommended for soil sampling for precision agriculture purposes. In this case, non-random samples on an established sampling grid replace the grouping of the landscape into similar areas. In this case, it is generally recommended that composite soil samples be taken on $15-30 \mathrm{~m}$ grids, which would be equivalent to 45 to 12 samples per ha. Soil sampling for $\mathrm{C}$ sequestration would face similar (or increased) spatial variability and therefore require a similar level of soil sampling intensity across the landscape. In estimating sampling costs, I considered a typical farm of 700 ha, with a sampling depth of $50 \mathrm{~cm}$ divided into at least six soil increments. While the cost of traditional soil sampling is negligible (absorbed by the farmer), this new mode of sampling for soil $\mathrm{C}$ would minimally cost $\$ 4.90 /$ sample, omitting overhead and maintenance expenses.

As with field sampling techniques, analysis of soil $\mathrm{C}$ in the laboratory will require additional considerations compared to traditional soil testing to address soil $\mathrm{C}$ sequestration. The most common method of testing soil for $\mathrm{C}$ or organic matter is the wet chemistry method of Walkley and Black or a method that measures weight loss on ignition. These methods are used because they are comparatively inexpensive, adaptive to large scale sample analysis, and results are sufficiently accurate for routine soil analysis. The Walkley and Black method measures oxidation of soil organic by dichromate $\left(\mathrm{Cr}_{2} \mathrm{O}_{7}\right) \mathrm{C}$ to estimate soil organic $\mathrm{C}$. However, only the easily oxidizable organic $\mathrm{C}$ is measured and a recovery factor of $77 \%$ is commonly used to convert "easily oxidizable" organic $\mathrm{C}$ to total organic $\mathrm{C}$. The Loss-on-Ignition method measures the change in soil weight (assumed to be the organic matter) before and after heating the soil to $360^{\circ} \mathrm{C}$. Estimates of the organic $\mathrm{C}$ are determined by regression analysis using selected soils covering a range in organic $\mathrm{C}$ expected in each testing area. This range is generally determined by the Walkley-Black method. The results from these tests, while being sufficient for discerning general soil trends or for determining pesticide application rates, are insufficiently precise and accurate for analyzing soil $\mathrm{C}$ sequestration (many laboratories publish a disclaimer explaining this point). An informal survey of U.S. soil testing laboratories found that the cost of organic matter analysis through these techniques ranged from $\$ 3.75$ to $\$ 16.00$ per sample, with a medium of $\$ 5.00$ per sample.

There is specialized laboratory equipment that will measure soil $\mathrm{C}$ with the precision and accuracy needed for $\mathrm{C}$ sequestration measurements. The most common of these utilizes combustion at $1000^{\circ} \mathrm{C}$ to break down samples to elemental forms and measures the resulting $\mathrm{CO}_{2}$ released. However, these instruments are expensive and costly to operate. In addition, sample preparation before analysis is increased, requiring additional fine grinding and precision weighing of the samples. This additional processing results in increased cost of analysis. The costs of laboratory preparation and minimum supplies for processing samples through a $\mathrm{C}$ analyzer were about $\$ 2.94 /$ sample, however, this does not include overhead costs of maintaining laboratory facilities. An informal survey of U.S. soil testing laboratories found that most did not offer $\mathrm{C}$ analysis. For those laboratories that did, costs varied widely, ranging from $\$ 3.75$ to $\$ 22.00 /$ sample, with a medium of $\$ 10.00 /$ sample.

Estimates of the cost of sampling a 700 ha farm were made using a minimum number of samples needed from methods of traditional sampling and the minimum cost of laboratory analysis. This meant that the farm was sampled by dividing the land area into 35 sections (20 ha/section). However, samples were also divided into 6 separate soil depths )or 210 samples) 
which would increase the sampling equipment needed and the time needed for sampling. Using the minimum estimates outlined above, the cost for sampling a 700 ha farm would be approximately $\$ 1650$.

In conclusion, soil sampling and analysis for $\mathrm{C}$ sequestration will require increased expense because it will require sampling of several soil depths and precision laboratory techniques. Inclusion of more soil depths will significantly increase sample numbers and sample collection expense. Precision laboratory techniques will significantly increase cost of equipment and sample processing. The cost was estimated at a minimum of $\$ 2.35$ per ha to develop a landscape data base that is reasonably reliable for $\mathrm{C}$ sequestration through standard soil testing techniques. 


\title{
Considerations and Procedures for Temporal Sampling of Soil Carbon
}

\author{
Ron Follett and John Kimble \\ USDA-ARS, Fort Collins CO, and Retired, USDA-NRCS
}

With the increasing interest in carbon sequestration in the soil, there is major concern about how to measure changes in carbon levels over short periods. Delegates at recent meeting in Montreal agreed that sequestration was a viable mechanism in reducing $\mathrm{CO}_{2}$ levels created by anthropogenic activities. If temporal changes are to be measured over a few years to decades, several factors and procedures must be considered, such as the differences in the soils' properties and how they may affect measurements. When looking at temporal changes, are the different organic- and inorganic- forms of carbon measured, and can the new in-situ equipment differentiate them? If assessments are to be accurate and representative of the soils, we need to know how different soils react to changes in carbon. For example, within Vertisols and Cryosols there is a natural mixing of the soil as it cracks periodically, allowing the SOC to penetrate deeply into the soils' profile. The following questions are among those that need to be answered: (1) Is a change occurring at all, and what is causing it ? (2) What is the rate of change and what affects this rate? (3) What is the rate of change in different $\mathrm{C}$ pools in the soil (active, slow release, and passive)? (4) How deep is the change occurring in the soils' profile? (5) Can the change be easily reversed? 


\section{APPENDIX C}

\section{Contributors and Attendees List}

\section{Galo Acosta}

Director of Research \& Development

AgCert, 1901 S. Harbor City Blvd

Melbourne, FL 32901

Tel: (321) 409-7840

E-mail: gacosta@agcert.com

\section{David J. Brown}

Asst. Professor of Landscape Pedology

Montana State University

Dept. of Land Resources \& Environmental

Sciences

334 Leon Johnson Hall, P.O. Box 173120

Tel: (406) 994-3724 (-3933 FAX)

E-mail: djbrown@montana.edu

\section{Nancy Cavallaro}

USDA-CSREES (Cooperative State Research, Education and Extension Service)

National Program Leader in Soils, Water, and Global Change

1400 Independence Ave. SW

Mail Stop 2241

Washington, DC 20024

Tel: (202) 401-5176; Fax: 202-401-6071

E-mail: ncavallaro@csrees.usda.gov

\section{Al Chan}

Sr. Scientist,

AgCert, 1901 S. Harbor City Blvd

Melbourne, FL 32901

Tel: (321) 409-7844

E-mail: achan@agcert.com

\section{Colin Christy}

Veris Technologies

601 N. Broadway

Salina, Kansas 67401

Tel: (785) 825 1851; E-mail;

christyc@veristech.com

\author{
Roger Dahlman, \\ DOE Office of Science, SC-74 \\ DOE/BER \\ 19901 Germantown Rd. \\ Germantown, MD 20874 \\ Tel: (301) 903-4951 \\ E-mail: Roger.Dahlman@science.doe.gov
}

\section{Paul Doraiswamy, USDA/ARS}

Hydrology and Remote Sensing Laboratory

Beltsville, MD 20706

Tel: (301) 504-6576

E-mail: pdoraiswamy@hydrolab.arsusda.gov

\section{Achim Dobermann}

Professor, Soil Science and Nutrient

Management Department of Agronomy \&

Horticulture University of Nebraska

253 Keim Hall, P.O. Box 830915

Lincoln, NE 68583-0915, USA

Tel: 1-402-472-1501; Fax: 1-402-472-7904

E-mail: adobermann2@unl.edu

http://agronomy.unl.edu;

http://soilfertility.unl.edu

\section{Oded Doron}

Brookhaven National Laboratory

Department Environmental Sciences

Bldg. 490D

Upton, NY 11973

Tel: (631) 344-3983

E-mail: odoron@bnl.gov

\section{Ben Ellert}

Environmental Health Department

Agricultural and Agri-Food Canada

Research Center

$5403-1^{\text {st }}$ Avenue South, P.O. Box 3000

Lethbridge, AB, Canada T1J4B1

Tel: (403) 317-2230

E-mail: ellert@agr.gc.ca 


\section{Ronny D. Harris}

Los Alamos National Laboratory

Earth \& Environmental Sciences Division

Atmospheric and Climate (EES-2)

P.O. Box 1663 MS J495

Los Alamos, NM 87545

Tel: (505) 665-9773

Fax: (505) 667-7460

rdharris@lanl.gov

\section{Cesar Izaurralde}

Pacific Northwest National Laboratory

Senior Research Scientist

Joint Global Change Research Institute

8400 Baltimore Ave. Suite 201

Collage Park, MD 20740

Tel: (301) 314-6751

E-mail: cesar.Izaurralde@pnl.gov

\section{Kurt Johnsen}

USDA Forest Service Southern Research

Station Biological Foundations of Southern

Forest Productivity and Sustainability

3041 Cornwallis Road

PO Box 12254

Research Triangle Park, NC 27709

Tel: (919) 549-4012

E-mail: kjohnsen@fs.fed.us

\section{John Kimble}

Consulting Research Soil Scientist

151 East Hill Church Road

Addison, NY 14801

Tel: (607) 3694475

E-mail: soilcarbon@aol.com

\section{Rattan Lal}

Professor

Ohio State University

Tel: (614) 292-9069

E-mail: 1al.1@osu.edu

\section{John Litynski}

Program Manager, DOE/FE/NETL

Tel: (304) 285-1339

E-mail: john.litynski@netl.doe.gov

\section{Kimberly A. Magrini-Bair}

National Renewable Energy Laboratory

1617 Cole Blvd.

Golden, CO 80401

Tel: (303) 384-7706

E-mail: kim_magrini@,nrel.gov

\section{Madhavi Martin}

Oak Ridge National Laboratory

P.O. Box 2008, MS-6038

Oak Ridge, TN 37831

Tel: (865) 574-7828

E-mail martinm1@ornl.gov

\section{Sudeep Mitra}

Brookhaven National Laboratory

Environmental Sciences Department

Upton, NY 11973

Tel: (631)344-6377

E-mail: smitra@bnl.gov

\section{Lisa M. Ramirez}

Brookhaven National Laboratory

Environmental Sciences Department

Tel: (631) 344-6377

E-mail: ramirezl@bnl.gov

\section{Aaron Rappaport}

Aaron Rappaport and Associates

15726 Piller Lane

Bowie, MD 20716

Tel: (301) 396-1835

E-mail: aaron_rappaport@,fastmail.fm

\section{James B. Reeves, III}

Research Chemist

USDA, Environ. Manag. and By-Products Lab.

ANRI, Building 306, RM 101

BARC East, Beltsville, MD 20705

Tel: (301) 504-8294;

E-mail: jreeves@nri.barc.usda.gov 
Chuck Rice

Kansas State University

Department of Agronomy

2701 Throckmorton

Manhattan, KS 66505

Tel: (785) 532 7217;

E-mail: cwrice@,ksu.edu

\section{Allison M. Thomson}

Senior Research Scientist

Joint Global Change Research Institute

8400 Baltimore Ave. Suite 201

Collage Park, MD 20740

Tel: (301) 314-6750

E-mail: allison.thomson@pnl.gov

\author{
Allen Torbert \\ Research Leader \\ USDA-ARS National Soil Dynamics Lab \\ 411 S. Donahue Dr. \\ Auburn, AL 36832-5806 \\ Tel: (334) 844-3979 \\ Fax: (334) 887-8597 \\ E-mail: atorbert@auburn.edu \\ Harold van Es \\ Professor of Soil and Water Management \\ Department of Crop and Soil Sciences \\ Cornell University, Ithaca, NY 14853-1901 \\ Tel: (607) 255-5629; \\ E-mail: hmv1@,cornell.edu

\section{Tristram O. West} \\ Environmental Sciences Division \\ Oak Ridge National Laboratory \\ P.O. Box 2008 \\ Oak Ridge, TN 37831-6335 \\ Tel: (865) 574-7322 \\ Fax: (865) 574-2232 \\ Email: westto@ornl.gov
}

\section{Lucian Wielopolski}

Scientist

Brookhaven National Laboratory

Environmental Science Department

Bldg. 490D

Upton, NY 11973

Tel: (631) 344-3656

E-mail: lwielo@bnl.gov

\section{Yue Zhang}

SUNY/Stony Brook

Applied Mathematics and Stat

Tel: (516) 383-1775

E-mail: sgnmm@ams.sunysb.edu

\section{Wei Zhu}

Associate Professor

Applied Mathematics and Stat

SUNY-SB

Tel: (631) 632-8374

E-mail: zhu@ams.sunysb.edu 


\section{APPENDIX D}

\section{Workshop PPT presentations}

included in the attached CD 